\title{
Häufigkeit endoprothetischer Hüft- und Knieoperationen
}

Florian Rothbauer, Ute Zerwes, Hans-Holger Bleß, Miriam Kip

2.1 Datenbasis -18

2.2 Inanspruchnahme Ersteingriffe - 21

2.3 Inanspruchnahme Wechseleingriffe und Revisionen - 23

2.4 Regionale Verteilung -25

2.5 Mengenentwicklung -29

2.5.1 Ersteingriffe -29

2.5.2 Wechseleingriffe und Revisionen - 31

2.6 Internationaler Vergleich -34

Literatur $\quad-41$ 


\section{Zusammenfassung}

Die jährliche Operationshäufigkeit endprothetischer Hüft- und Knieersteingriffe in der Bevölkerung ist stabil und hat seit 2007 nicht zugenommen. Sie beträgt für Hüftersteingriffe in der Altersgruppe der über 70-Jährigen 1,1\% (2007 und 2014) und für Knieersteingriffe 0,7\% (2007) bzw. 0,6\% (2014). Die Operationshäufigkeit bezogen auf die Gesamtbevölkerung betrug 2014 0,26\% (Hüfte) bzw. 0,19\% (Knie). In Deutschland waren 2014 rund 219.000 Hüft- und rund 149.000 Knieersteingriffe dokumentiert. Am häufigsten wird ein Totalersatz des entsprechenden Gelenkes vorgenommen. Rund $40 \%$ der endoprothetischen Hüft- oder Knieersteingriffe fallen in die Altersgruppe 70-79 Jahre; Frauen sind häufiger betroffen als Männer (Verhältnis 2:1). Die absolute Anzahl vorgenommener Wechseleingriffe (inklusive Revisionen ohne Wechsel) betrug 2014 rund 30.000 (Hüfte) bzw. 20.000 (Knie). Die Anzahl der Wechseleingriffe eines Jahres steht nicht zwangsläufig in direktem Bezug zu den Ersteingriffen desselben Jahres. Die Anzahl der Wechseleingriffe ist vielmehr in Relation zur kumulierten Anzahl an endoprothetischen Ersteingriffen der letzten Jahre und Jahrzehnte zu sehen. Wie bei den Ersteingriffen fallen rund $40 \%$ der Wechseleingriffe in die Altersgruppe der 70- bis 79-Jährigen; der Geschlechterunterschied ist aber im Vergleich weniger deutlich ausgeprägt.

Auch die Operationshäufigkeit vorgenommener Hüft- und Kniewechseleingriffe (inklusive Revisionen ohne Wechsel) hat bezogen auf die Bevölkerung im Zeitraum 2007-2014 nicht zugenommen. Bei den über 70-Jährigen betrug die Häufigkeit vorgenommener Wechseleingriffe (inklusive Revisionen ohne Wechsel) in 2014 0,19\% (Hüfte) bzw. 0,10 \% (Knie). Die jährliche Inanspruchnahme endoprothetischer Hüft- und Knieersteingriffe variiert international. Auch innerhalb Deutschlands gibt es regionale Unterschiede, wie Auswertungen der gesetzlichen Krankenversicherung aus dem Zeitraum 2005-2011 ergaben. Eine vergleichsweise geringe Inanspruchnahme war insbesondere mit einem niedrigen Vorkommen der Arthrose, einem niedrigen Sozialstatus, einer hohen Facharztdichte (Orthopäde) und dem Wohnort des Patienten im städtischen Raum assoziiert.
Die endoprothetische Hüft- oder Knieoperation ist eine wirksame Therapie für Patienten mit erheblicher (drohender), dauerhafter eingeschränkter Funktionalität des Gelenkes aufgrund von Destruktion und Schmerzen, die anders nicht mehr behandelbar sind, sowie für die Behandlung gelenknaher Frakturen. Die verschiedenen Gelenkersatzverfahren sollen eine gute Funktionalität, Belastbarkeit und Lebensqualität wiederherstellen. Die Betrachtung der Häufigkeit (Inanspruchnahme) ist dabei eine wichtige Komponente für die Versorgungsplanung im ambulanten und stationären Bereich, zur Abschätzung des Bedarfs und Folgebedarfs z. B. an rehabilitativen Maßnahmen und Allokationsfragen. Das folgende Kapitel stellt die Inanspruchnahme von Hüft- und Kniegelenkersatz insgesamt und differenziert nach Alter und Geschlecht sowie nach Art und Verankerungstechnik in Deutschland dar. Die Darstellung unterscheidet zwischen Erst- und Wechseleingriffen. Des Weiteren wird auf regionale Verteilungsunterschiede und die zeitliche Entwicklung der Inanspruchnahme in Deutschland und im internationalen Vergleich eingegangen.

\subsection{Datenbasis}

Die Klassifikation nach dem Operationen- und Prozedurenschlüssel (OPS) ermöglicht für die stationäre Versorgung eine differenzierte Betrachtung der jährlich erbrachten Erstoperationen und Wechseloperationen von Hüft- und Kniegelenkersatzverfahren in Deutschland. Der OPS wird im deutschen Gesundheitssystem in erster Linie zu administrativen Zwecken für die Identifikation von im Krankenhaus am Patienten erbrachten Leistungen verwendet.

Im Abschnitt 5-82 des OPS ist der Knochenund Gelenkersatz klassifiziert (• Tab. 2.1). Die Kodiersystematik erlaubt zuverlässig eine Unterscheidung zwischen Ersteingriff, Revision, Wechsel oder Entfernung von Hüftgelenken (5-820/5-821) und Kniegelenken (5-822/5-823). Weiterhin sind das Alter und das Geschlecht der Patienten ausgewiesen. Die OPS 5-820 und 5-822 dokumentieren die erstmalige endoprothetische Versorgung (Ersteingriffe) des Hüft- bzw. des Kniegelenkes. Die OPS 5-821 bzw. 5-823 und deren weiter differenzierende 
- Tab. 2.1 Klassifikation gemäß OPS

\begin{tabular}{|c|c|c|c|}
\hline OPS & Beschreibung & OPS & Beschreibung \\
\hline \multicolumn{4}{|c|}{ Hüfte: Ersteingriff } \\
\hline $5-820.0$ & Totalendoprothese & $5-820.2$ & Totalendoprothese, Sonderprothese \\
\hline $5-820.3$ & Femurkopfprothese & $5-820.4$ & Duokopfprothese \\
\hline $5-820.5$ & Gelenkpfannenstützschale & $5-820.7$ & Gelenkschnapp-Pfanne \\
\hline $5-820.8$ & Oberflächenersatzprothese & $5-820.9$ & Kurzschaft-Femurkopfprothese \\
\hline $5-820 . x$ & Sonstige & 5-820.y & N. n. bez. \\
\hline \multicolumn{4}{|c|}{ Hüfte: Wechseleingriffe und Revision } \\
\hline $5-821.0$ & Revision (ohne Wechsel) & $5-821.1$ & Wechsel einer Femurkopfprothese \\
\hline $5-821.2$ & Wechsel einer Gelenkpfannenprothese & $5-821.3$ & $\begin{array}{l}\text { Wechsel einer zementierten } \\
\text { Totalendoprothese }\end{array}$ \\
\hline $5-821.4$ & $\begin{array}{l}\text { Wechsel einer nichtzementierten } \\
\text { Totalendoprothese }\end{array}$ & 5-821.5 & Wechsel einer Totalendoprothese, hybrid \\
\hline $5-821.6$ & $\begin{array}{l}\text { Wechsel einer Totalendoprothese, } \\
\text { Sonderprothese }\end{array}$ & $5-821.7$ & Entfernung einer Totalendoprothese \\
\hline $5-821.8$ & Entfernung einer Femurkopfprothese & $5-821.9$ & Entfernung einer Duokopfprothese \\
\hline 5-821.a & Entfernung einer Femurkopfkappe & $5-821 . b$ & Entfernung einer Gelenkpfannenprothese \\
\hline 5-821.c & $\begin{array}{l}\text { Entfernung einer Gelenkpfannenstütz- } \\
\text { schale }\end{array}$ & 5-821.d & Entfernung einer Gelenkschnapp-Pfanne \\
\hline 5-821.e & $\begin{array}{l}\text { Entfernung einer Totalendoprothese, } \\
\text { Sonderprothese }\end{array}$ & 5-821.f & Wechsel einer Duokopfprothese \\
\hline $5-821.9$ & Wechsel einer Oberflächenersatzprothese & 5-821.h & Entfernung einer Oberflächenersatzprothese \\
\hline 5-821.j & $\begin{array}{l}\text { Wechsel einer schenkelhalserhaltenden } \\
\text { Femurkopfprothese (Kurzschaft-Femur- } \\
\text { kopfprothese) }\end{array}$ & 5-821.k & $\begin{array}{l}\text { Entfernung einer schenkelhalserhaltenden } \\
\text { Femurkopfprothese (Kurzschaft-Femurkopf- } \\
\text { prothese) }\end{array}$ \\
\hline $5-821 . x$ & Sonstige & 5-821.y & N. n. bez. \\
\hline
\end{tabular}

Knie: Ersteingriff

\begin{tabular}{|c|c|c|c|}
\hline $5-822.0$ & Unikondyläre Schlittenprothese & $5-822.1$ & $\begin{array}{l}\text { Bikondyläre Oberflächenersatzprothese, } \\
\text { ungekoppelt, ohne Patellaersatz }\end{array}$ \\
\hline $5-822.2$ & $\begin{array}{l}\text { Bikondyläre Oberflächenersatzprothese, } \\
\text { ungekoppelt, mit Patellaersatz }\end{array}$ & $5-822.3$ & $\begin{array}{l}\text { Bikondyläre Oberflächenersatzprothese, } \\
\text { teilgekoppelt, ohne Patellaersatz }\end{array}$ \\
\hline $5-822.4$ & $\begin{array}{l}\text { Bikondyläre Oberflächenersatzprothese, } \\
\text { teilgekoppelt, ohne Patellaersatz }\end{array}$ & $5-822.6$ & Scharnierendoprothese ohne Patellaersatz \\
\hline $5-822.7$ & Scharnierendoprothese mit Patellaersatz & $5-822.8$ & Patellaersatz \\
\hline $5-822.9$ & Sonderprothese & 5-822.a & $\begin{array}{l}\text { Endoprothese mit erweiterter Beugefähig- } \\
\text { keit, ohne Patellaersatz }\end{array}$ \\
\hline 5-822.b & $\begin{array}{l}\text { Endoprothese mit erweiterter Beugefähig- } \\
\text { keit, mit Patellaersatz }\end{array}$ & 5-822.c & Interpositionelles nicht verankertes Implantat \\
\hline 5-822.d & $\begin{array}{l}\text { Bikompartimentelle Teilgelenkersatz- } \\
\text { prothese, ohne Patellaersatz }\end{array}$ & 5-822.e & $\begin{array}{l}\text { Bikompartimentelle Teilgelenkersatzprothese, } \\
\text { mit Patellaersatz }\end{array}$ \\
\hline
\end{tabular}


- Tab. 2.1 (Fortsetzung)

\begin{tabular}{|c|c|c|c|}
\hline OPS & Beschreibung & OPS & Beschreibung \\
\hline 5-822.f & $\begin{array}{l}\text { Implantation eines endoprothetischen } \\
\text { Gelenkersatzes ohne Bewegungsfunktion }\end{array}$ & $5-822 \cdot x$ & Sonstige \\
\hline 5-822.y & N. n. bez. & & \\
\hline \multicolumn{4}{|c|}{ Knie: Revision und Wechseleingriff } \\
\hline $5-823.0$ & Revision (ohne Wechsel) & $5-832.1$ & $\begin{array}{l}\text { Wechsel einer unikondylären Schlitten- } \\
\text { prothese }\end{array}$ \\
\hline $5-823.2$ & $\begin{array}{l}\text { Wechsel einer bikondylären Schlitten- } \\
\text { prothese }\end{array}$ & $5-823.3$ & Wechsel einer Scharnierendoprothese \\
\hline $5-823.4$ & Wechsel einer Sonderprothese & $5-823.5$ & Wechsel eines Patellaersatzes \\
\hline $5-823.6$ & $\begin{array}{l}\text { Entfernung einer unikondylären Schlitten- } \\
\text { prothese }\end{array}$ & $5-823.7$ & $\begin{array}{l}\text { Entfernung einer bikondylären Oberflächen- } \\
\text { ersatzprothese }\end{array}$ \\
\hline $5-823.8$ & Entfernung einer Scharnierendoprothese & $5-823.9$ & Entfernung eines Patellaersatzes \\
\hline 5-823.a & Entfernung einer Sonderprothese & 5-823.b & $\begin{array}{l}\text { Wechsel einer Endoprothese mit erweiterter } \\
\text { Beugefähigkeit }\end{array}$ \\
\hline 5-823.c & $\begin{array}{l}\text { Wechsel eines interpositionellen nicht } \\
\text { verankerten Implantates }\end{array}$ & 5-823.d & $\begin{array}{l}\text { Entfernung einer Endoprothese } \\
\text { mit erweiterter Beugefähigkeit }\end{array}$ \\
\hline 5-823.e & $\begin{array}{l}\text { Entfernung eines interpositionellen nicht } \\
\text { verankerten Implantates }\end{array}$ & $5-823 . f$ & $\begin{array}{l}\text { Wechsel einer bikompartimentellen } \\
\text { Teilgelenkersatzprothese }\end{array}$ \\
\hline 5-823.g & $\begin{array}{l}\text { Entfernung einer bikompartimentellen } \\
\text { Teilgelenkersatzprothese }\end{array}$ & $5-823 . h$ & $\begin{array}{l}\text { Wechsel eines endoprothetischen } \\
\text { Gelenkersatzes ohne Bewegungsfunktion }\end{array}$ \\
\hline 5-823.j & $\begin{array}{l}\text { Entfernung eines endoprothetischen } \\
\text { Gelenkersatzes ohne Bewegungsfunktion }\end{array}$ & $5-823 . x$ & Sonstige \\
\hline 5-823.y & N. n. bez. & & \\
\hline
\end{tabular}

Unterschlüssel setzen einen vorausgehenden endoprothetischen Eingriff am selben Gelenk voraus, bezeichnen also die Wechseleingriffe und Revisionen (Zweit- oder Folgeeingriffe).

Das Statistische Bundesamt macht die Nutzung der OPS gemäß $\$ 21$ des Gesetzes über die Entgelte für voll- und teilstationäre Krankenhausleistungen öffentlich zugänglich. Die Daten können nur fallbezogen und nicht patientenbezogen abgerufen werden, d. h., die Anzahl der Fälle entspricht nicht (unbedingt) der Anzahl der Patienten. Zweizeitig durchgeführte Eingriffe werden dabei als zwei Fälle dokumentiert, mit der Folge von Mehrfachzählungen einzelner Patienten.

Nicht möglich ist die statistische Auswertung des operativen Zugangs, des verwendeten Endopro- thesenmaterials oder ob ein Eingriff geplant oder als Notfall durchgeführt werden musste. Ebenfalls nicht möglich ist die Ermittlung der Haltbarkeit von Endoprothesen (Standzeit) aufgrund der Daten des Statistischen Bundesamtes, da zwischen dem Einund Ausbau der Endoprothese beim einzelnen Patienten kein Zusammenhang hergestellt werden kann. Auch eine Verbindung zu der zugrunde liegenden Indikation (Arthrose, Frakturen, andere Ursachen) kann auf Basis des Datensatzes des Statistischen Bundesamtes nicht abgebildet werden. Zwar wird eine Kopplung von Diagnose und Prozedur grundsätzlich von den Krankenhäusern an die Krankenkassen sowie das Institut für das Entgeltsystem im Krankenhaus (InEK) gemeldet, öffentlich ist die Kombination dieser Daten jedoch nicht 


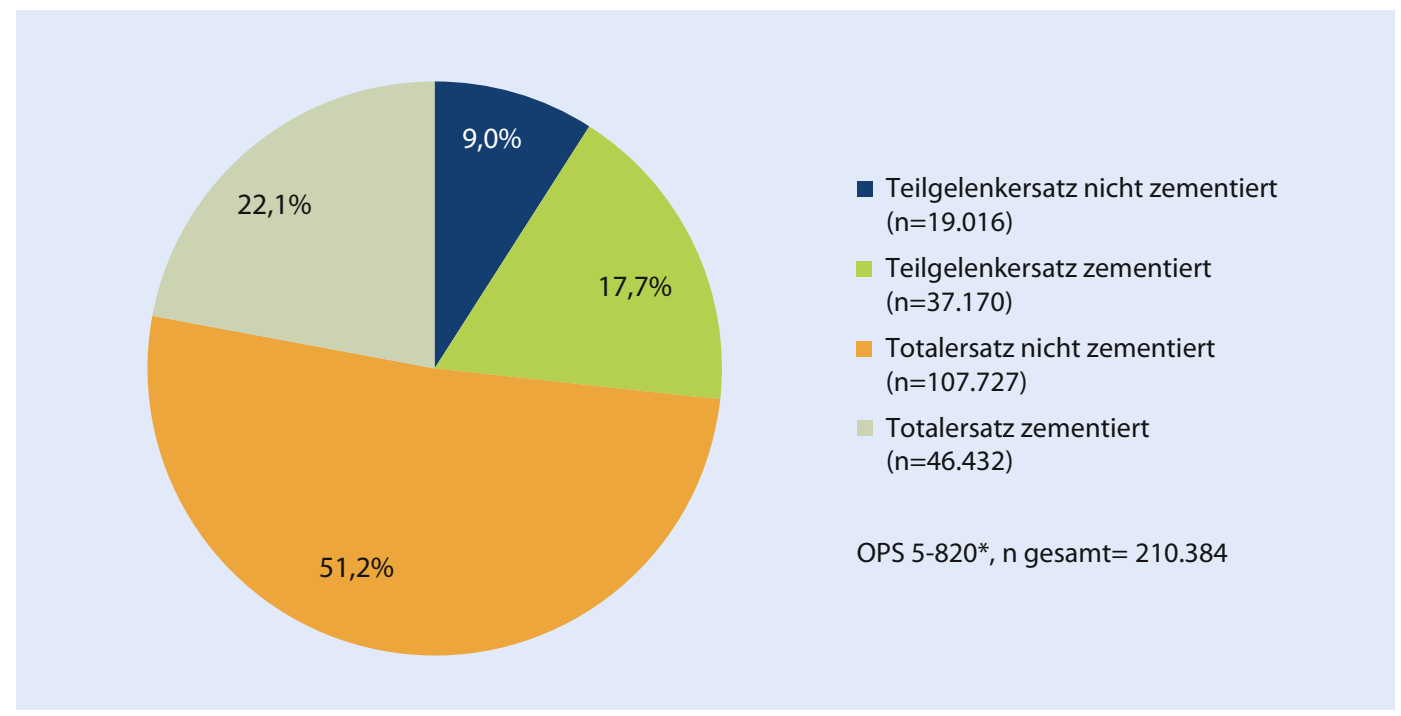

- Abb. 2.1 Verteilung der Inanspruchnahmen ( $n=210.384)$ eines Hüftgelenkersatzes (OPS 5-820.*) differenziert nach Totalund Teilersatz und Verankerung (2013). (IGES - Statistisches Bundesamt 2014)

zugänglich. Des Weiteren sind andere für die Beschreibung der Indikation notwendige klinische Parameter wie Schmerzen, Funktionalität oder Lebensqualität nicht abgebildet. Auswertungen des deutschen Endoprothesenregisters (EPRD) können in Zukunft Aufschluss über diese Zusammenhänge (z. B. Indikation und Prozedur) geben ( $\triangleright$ Kap. 4). Da das Risiko für einen Gelenkersatz nicht gleichermaßen über alle Bevölkerungs- und Altersgruppen verteilt ist, gilt, dass belastbare Aussagen zu Unterschieden in der Häufigkeit (z. B. bei regionalen oder internationalen vergleichenden Betrachtungen) nur nach Adjustierung oder Standardisierung der jeweiligen Datenbasis hinsichtlich beeinflussender Merkmale, wie z. B. Alter und Geschlecht, vorgenommen werden können. Regionale Auswertungen von Krankenkassendaten (z. B. Schäfer et al. 2013; Lüring et al. 2013) berichten in der Regel nach der Bevölkerungsstruktur standardisierte Häufigkeiten. Des Weiteren sollte bei vergleichenden Betrachtungen die gleiche Erhebungsmethodik Anwendung finden, um gut belastbare Aussagen machen zu können. In Darstellungen patientenbezogener Daten der OECD zur Häufigkeit endoprothetischer Hüft- und Knieoperationen im internationalen Vergleich sind diese Aspekte in aller Regel nicht ausreichend berücksichtigt ( $\triangleright$ Kap. 6).

\subsection{Inanspruchnahme Ersteingriffe}

Ausgehend von Daten des Statistischen Bundesamtes wurden im Jahr 2014 insgesamt 219.325 und in 2013 insgesamt 210.384 endoprothetische Hüftersteingriffe durchgeführt (absolute Anzahl). Von den in 2013210.384 vorgenommenen Hüftersteingriffen waren 154.159 (73,3\%) Totalendoprothesen (TEP) und 56.225 (26,7\%) Teilendoprothesen. 60,2\% (126.743 Fälle) aller Hüftendoprothesen wurden zementfrei implantiert (Statistisches Bundesamt 2014) (• Abb. 2.1). Bezogen auf den Bevölkerungsstand (Stichtag 31.12.2014) betrug die OPHäufigkeit in 2014 0,26 \% (eigene Berechnung, Statistisches Bundesamt 2014, Statistisches Bundesamt 2015).

Die absolute Anzahl der Ersteingriffe am Knie betrug im Jahr 2014149.126 und in 2013 143.024. Von den in 2013 durchgeführten 143.024 durchgeführten Knieersteingriffen waren 84\% der Eingriffe ein bikondylärer Ersatz (• Abb. 2.2). Die OP-Häufigkeit bezogen auf die Gesamtbevölkerung (Bevölkerungsstand 2014, Stichtag 31.12.2014) lag beim Kniegelenkersatz bei 0,19\% in 2014 (eigene Berechnung, Statistisches Bundesamt 2014, Statistisches Bundesamt 2015). Im Gegensatz zu den Ersteingriffen der Hüfte wurde beim Knie bei einem Großteil 


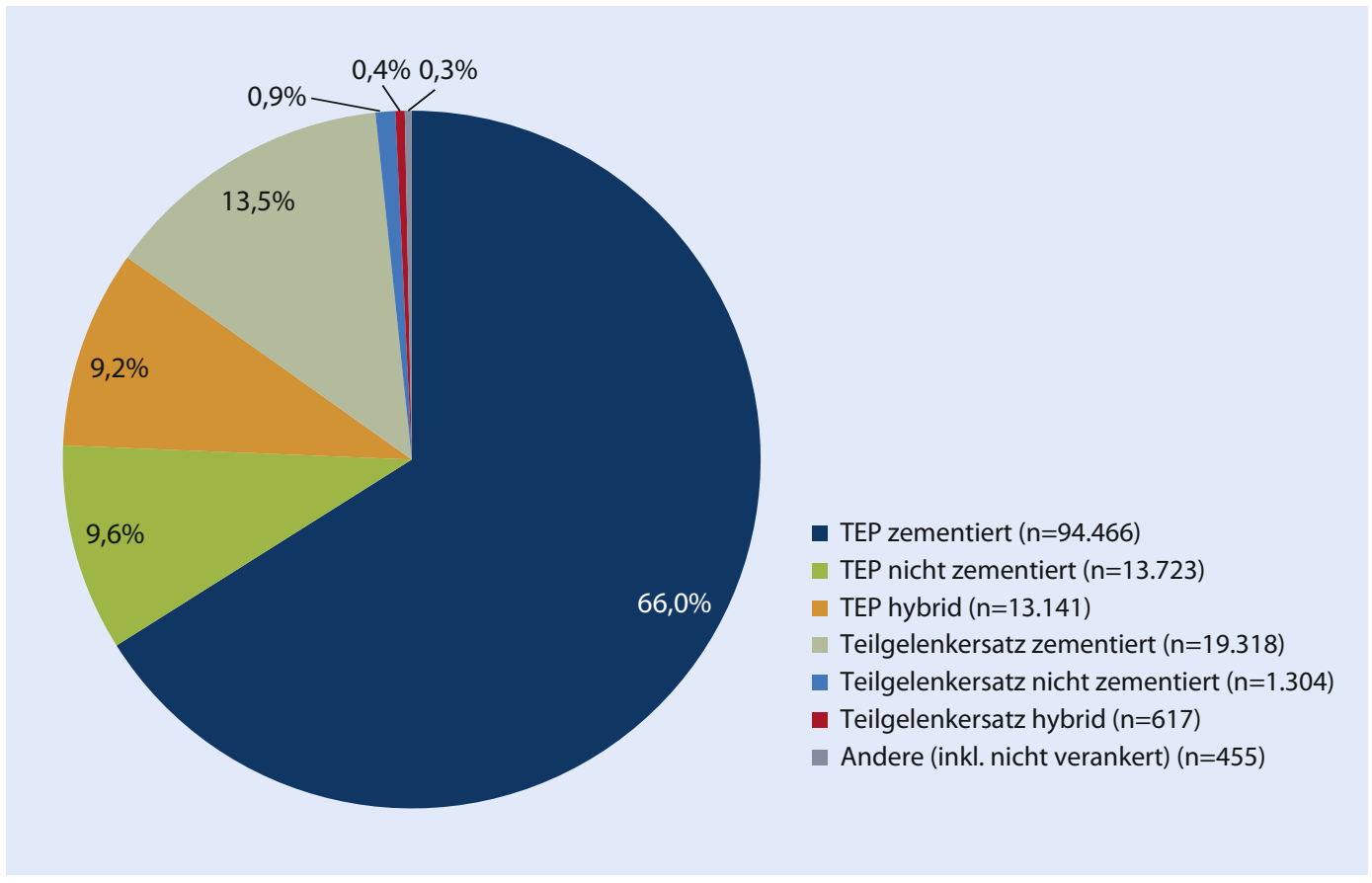

- Abb. 2.2 Verteilung der Inanspruchnahme (absolute Anzahl, $n=143.024$ ) endoprothetischer Knieersteingriffe (OPS 5-822.*) differenziert nach Total- und Teilersatz und Verankerung (2013). (IGES - Statistisches Bundesamt 2014)

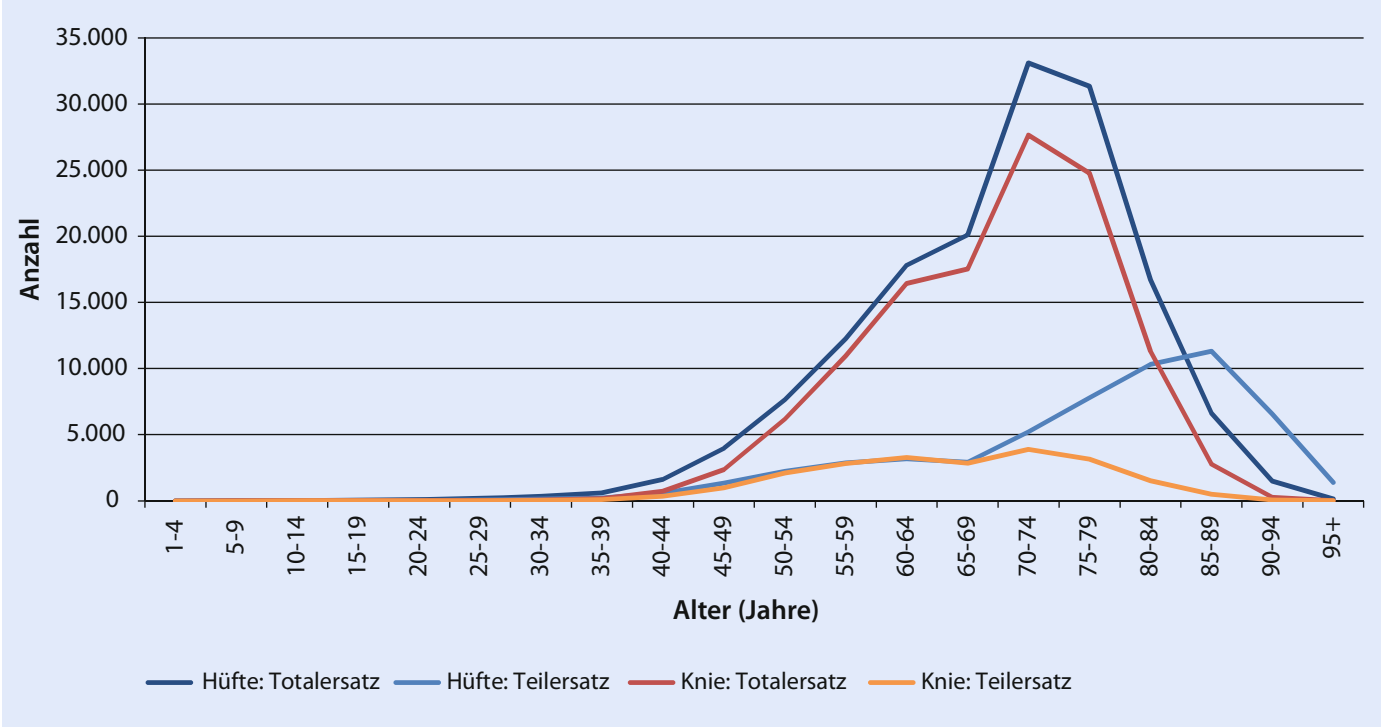

- Abb. 2.3 Inanspruchnahme (absolute Anzahl) von endoprothetischen Hüft- und Knieersteingriffen differenziert nach Total- und Teilersatz und Altersgruppen (2013). (IGES - Statistisches Bundesamt 2014) 
der Erstoperationen (79,6\%) eine zementierte Verankerung des Gelenkersatzes durchgeführt. Eine komplett zementfreie Verankerung war bei 10,5\% und die hybride/teilzementierte Verankerung bei 9,6\% der Eingriffe dokumentiert (Statistisches Bundesamt 2014).

Gut $65 \%$ der Ersteingriffe an der Hüfte oder am Knie in der Bevölkerung über 60 Jahre betrafen Frauen (Statistisches Bundesamt 2014). Ein höherer Anteil an weiblichen Patienten in der Hüft- und Knieendoprothetik ist auch an anderer Stelle gut dokumentiert (Braun 2013; Lüring et al. 2013). Bedingt ist der höhere Anteil an weiblichen Patienten unter anderem durch die höhere Prävalenz der Arthrose (häufigste Indikation für einen endoprothetischen Hüft- oder Knieeingriff) sowie die signifikant höhere Lebenserwartung von Frauen (Rabenberg 2013).

Die Inanspruchnahme der Ersteingriffe ist deutlich mit dem Alter der Patienten assoziiert: Rund $40 \%$ der in Deutschland dokumentierten endoprothetischen Hüft- oder Knieersteingriffe fallen in die Altersgruppe 70-79 Jahre (• Abb. 2.3). Das Durchschnittsalter bei Erstoperationen einer Hüftoder Knie-TEP betrug 2013 69,7 bzw. 69,2 Jahre. Patienten, die einen Teilgelenkersatz am Knie erhielten, waren im Durchschnitt etwas jünger
(Durchschnittsalter 65,8 Jahre). Die Anzahl der Patienten, die einen Teilgelenkersatz der Hüfte erhalten, ist wiederum unter den 85 - bis 89 -Jährigen am höchsten. In dieser Altersgruppe sind insgesamt mehr Fälle von Hüftersteingriffen mit Teilendoprothesen im Vergleich zu Totalendoprothesen dokumentiert. Dies ist vor allen Dingen in der hohen Prävalenz von Schenkelhalsfrakturen begründet, die in dieser Altersgruppe besonders häufig vorkommen und hauptsächlich mit Teilendoprothesen versorgt werden ( $\triangleright$ Abschn. 1.2.1 und $\triangleright$ Abschn. 1.2.2) (• Abb. 2.3) (Statistisches Bundesamt 2014).

Auch hinsichtlich der Verankerungstechnik ist ein Zusammenhang zum Alter der Patienten zu beobachten: Der Anteil an Hüft-TEP mit Zementierungen im Vergleich zu Hüft-TEP ohne Zementierung nimmt mit zunehmendem Alter der Patienten zu (Statistisches Bundesamt 2014).

\subsection{Inanspruchnahme Wechsel- eingriffe und Revisionen ohne Wechsel}

Gemäß Statistischem Bundesamt wurden in 2014 insgesamt 35.133 und in 2013 insgesamt 31.067

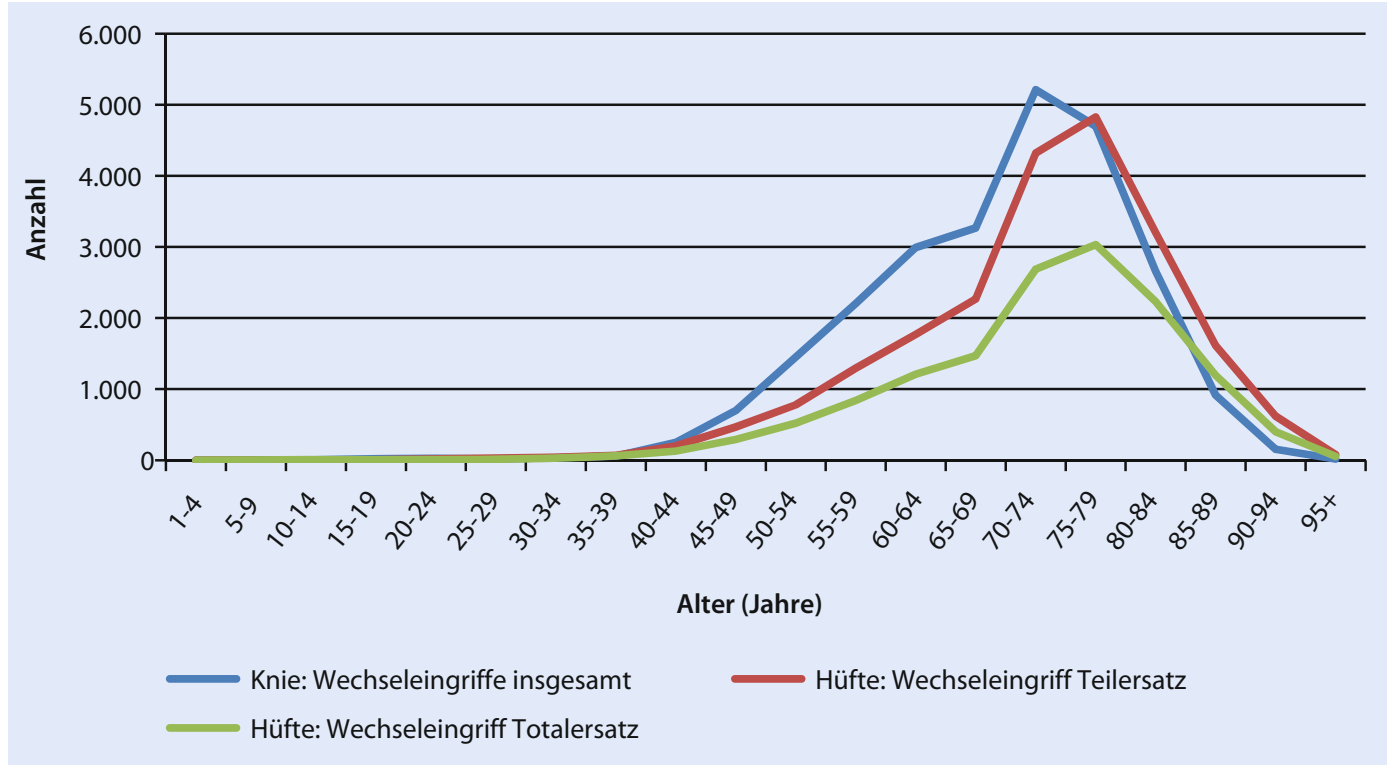

- Abb. 2.4 Inanspruchnahme (absolute Anzahl) von Wechseleingriffen inklusive Revisionen ohne Wechsel differenziert nach Art und Altersgruppe (2013). (Quelle: IGES - Statistisches Bundesamt 2014) 
Hüft- und 21.678 Kniewechseleingriffe (inklusive Revisionen ohne Wechsel) durchgeführt (absolute Anzahl). Bezogen auf den Bevölkerungsstand (Stichtag 31.12.2014), entsprach dies einer OP-Häufigkeit von 0,04 \% (Hüfte) bzw. 0,06 \% (Knie) in 2014 (eigene Berechnung, Statistisches Bundesamt 2014, Statistisches Bundesamt 2015). 3.784 Fälle bzw. 3.213 der Fälle in 2013 waren Revisionen ohne Wechsel von Teilen an der Hüfte bzw. am Knie. Revisionen ohne Wechsel machten somit rund $12 \%$ bzw. $16 \%$ aller dokumentierten Hüft- bzw. Kniewechseleingriffe eines Jahres (2013) aus. Der Ersatz einer Gelenkpfannenprothese (Teilersatz) bzw. die bikondyläre Oberflächenersatzprothese waren die am häufigsten durchgeführten Wechseleingriffe am Hüft- bzw. Kniegelenk (• Tab. 2.2) (Statistisches Bundesamt 2014).

Die höchste Anzahl durchgeführter Wechseleingriffe und Revisionen (Total- und Teilersatz) betrifft in 2013 die Altersgruppe 75-79 Jahre. $40 \%$ aller Wechseleingriffe und Revisionen an der Hüfte und am Knie betreffen die Altersgruppe 70-79 Jahre. 2013 lag das Durchschnittsalter der Patienten bei Wechseleingriffen und Revisionen der Hüfte bei 72,5 Jahren und des Knies bei 69 Jahren und ist dabei etwas höher als das Durchschnittsalter der Patienten bei Ersteingriffen (• Abb. 2.4) (Statistisches Bundesamt 2014).

Die absolute Anzahl für Wechsel- und Revisionseingriffe liegt wie für Ersteingriffe bei Frauen über der bei Männern. Wenn man beachtet, dass die absolute Häufigkeit der Ersteingriffe bei Männern im Vergleich zu Frauen deutlich geringer ausfällt, wird bei Männern vergleichsweise häufiger eine Revision- oder ein Wechseleingriff durchgeführt (- Abb. 2.5).

Ein direkter Bezug der in Anspruch genommenen Wechseleingriffe zu den Ersteingriffen desselben Jahres kann allerdings nicht hergestellt werden; aufgrund der mittleren Standzeiten der Endoprothesen ist die Anzahl der Wechseleingriffe in Relation zur kumulierten Anzahl an Ersteingriffen der letzten Jahre und Jahrzehnte zu setzen. Aspekte zur Bewertung der Häufigkeit von Wechseleingriffen (inklusive Revisionen ohne Wechsel) werden im Expertenkapitel ausgeführt ( $\triangleright$ Kap. 6).

- Tab. 2.2 Inanspruchnahme (absolute Anzahl) von Wechseleingriffen und Revisionen am Hüft- und Kniegelenk (2013)

Beschreibung

Hüftgelenk

Totalersatz

Wechsel Totalendoprothese (nichtzementiert)

Wechsel Totalendoprothese (zementiert)

Wechsel Totalendoprothese

(teilzementiert)

Wechsel Sonderprothese

Teilersatz

Wechsel Gelenkpfannenprothese

Wechsel Femurkopfprothese

Wechsel Duokopfprothese

Wechsel Oberflächenersatz-

prothese

Wechsel schenkelhalserhaltende

Femurkopfprothese

Revision (ohne Wechsel)

3.784

Wechseleingriffe und Revisionen

Gesamt

\section{Kniegelenk}

bikondyläre Oberflächenersatz-

prothese

Wechsel unikondyläre Schlitten-

prothese

Wechsel Scharnierendoprothese

Wechsel Endoprothese mit erweiterter Beugefähigkeit

Wechsel Sonderprothese

Wechsel bikompartimentelle

Teilgelenkersatzprothese

Wechsel Patellaersatz

Andere

Revision (ohne Wechsel)

$\%$

40,1

15,6

3,0

0,7

Gesamt

100 


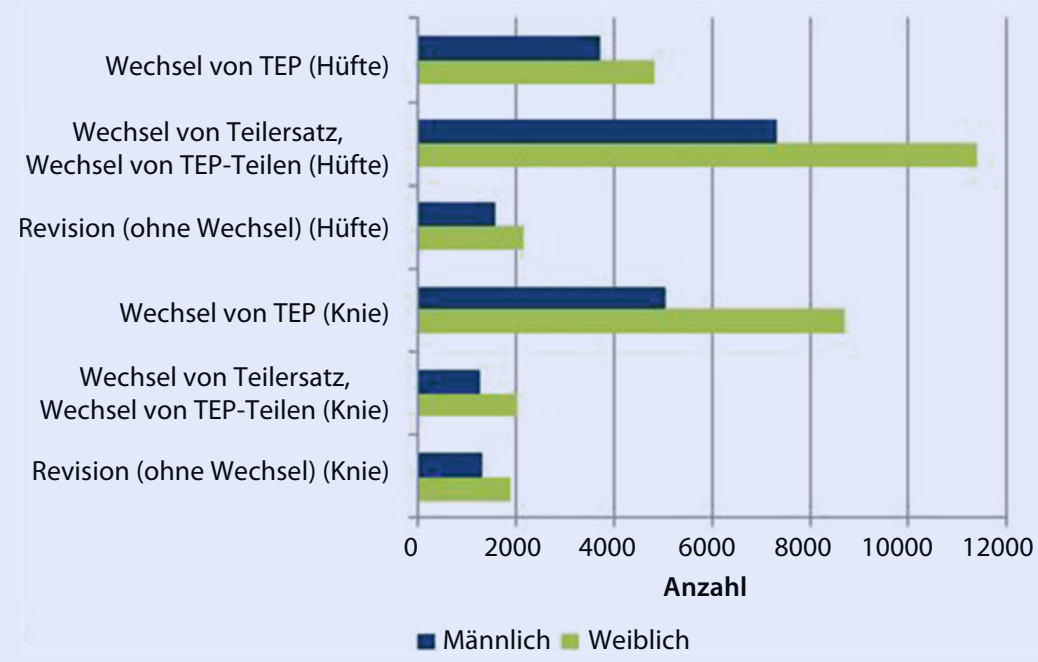

- Abb. 2.5 Inanspruchnahme (absolute Anzahl) von Gelenkersatzverfahren am Hüft- und Kniegelenk differenziert nach Wechseleingriffen inklusive Revisionen ohne Wechsel und Geschlecht (2013). (IGES - Statistisches Bundesamt 2014)

\subsection{Regionale Verteilung}

Auf Basis von Abrechnungsdaten (Sekundärdaten) von AOK-Versicherten unter Einbezug von 24 Millionen Versicherten der Jahre 2005-2009 wurde die regionale Verteilung in der Hüft- und Kniegelenkersatzversorgung von Schäfer et al. auf Länderund Kreisebene untersucht. Die Autoren errechneten altersstandardisierte Operationsraten (Ersteingriffe an der Hüfte und am Knie bezogen auf 100.000 Versicherte pro Jahr). Es wurden ausschließlich TEP berücksichtigt. Die Raten wurden altersstandardisiert (Europastandard) errechnet, um Verzerrungen aufgrund demographischer Unterschiede zwischen den Regionen zu minimieren und um die Ergebnisse regional und mit anderen Studien mit demselben Vergleichsstandard vergleichbar zu machen (Schäfer et al. 2013).

Insgesamt wurden 2009148 Hüft- und 132 Knieerstoperationen pro 100.000 AOK-Versicherten durchgeführt. Auf der Ebene der Bundesländer zeigten sich hier deutliche Unterschiede: Die niedrigste Eingriffsrate an der Hüfte wurde in Berlin mit 120 und die höchste in Niedersachsen mit 168 dokumentiert. Dies entspricht einem Unterschied von ca. $40 \%$ (• Abb. 2.6). Die Eingriffsraten am Knie zeigten ebenfalls deutliche regionale Schwankungen auf Länderebene (78,4\%): Die niedrigste Eingriffsrate war ebenfalls in Berlin zu beobachten (90). Am häufigsten wurde in der Studienpopulation eine KnieTEP in Bayern erstmalig eingesetzt (160). Werden nach Ausschluss der Stadtstaaten nur die Flächenländer betrachtet, liegen die niedrigsten Eingriffsraten an der Hüfte in Sachsen-Anhalt (143) und am Knie in Mecklenburg-Vorpommern (109) und die höchsten in Bayern, Niedersachsen und SchleswigHolstein bzw. Thüringen (Schäfer et al. 2013).

Auf Kreisebene zeigten sich anhand der AOKErhebung ebenfalls große Unterschiede. Die niedrigste Eingriffsrate an der Hüfte (gemittelter Wert für den Zeitraum 2005 bis 2009) lag bei 106 Fällen (Kreis Neustadt an der Weinstraße), der höchste Wert lag bei 216 Fällen pro 100.000 Versicherte (Neustadt an der Aisch). Auch auf Kreisebene sind die regionalen Unterschiede in der Inanspruchnahme von TEP am Knie größer ausgeprägt als bei den Hüftverfahren (Schäfer et al. 2013).

Ein Report der Deutschen Gesellschaft für Orthopädie und Orthopädische Chirurgie im Auftrag der Bertelsmann Stiftung zu regionalen Unterschieden und deren Einflussfaktoren mit dem Schwerpunkt Knieendoprothetik berichtete ebenfalls deut- 


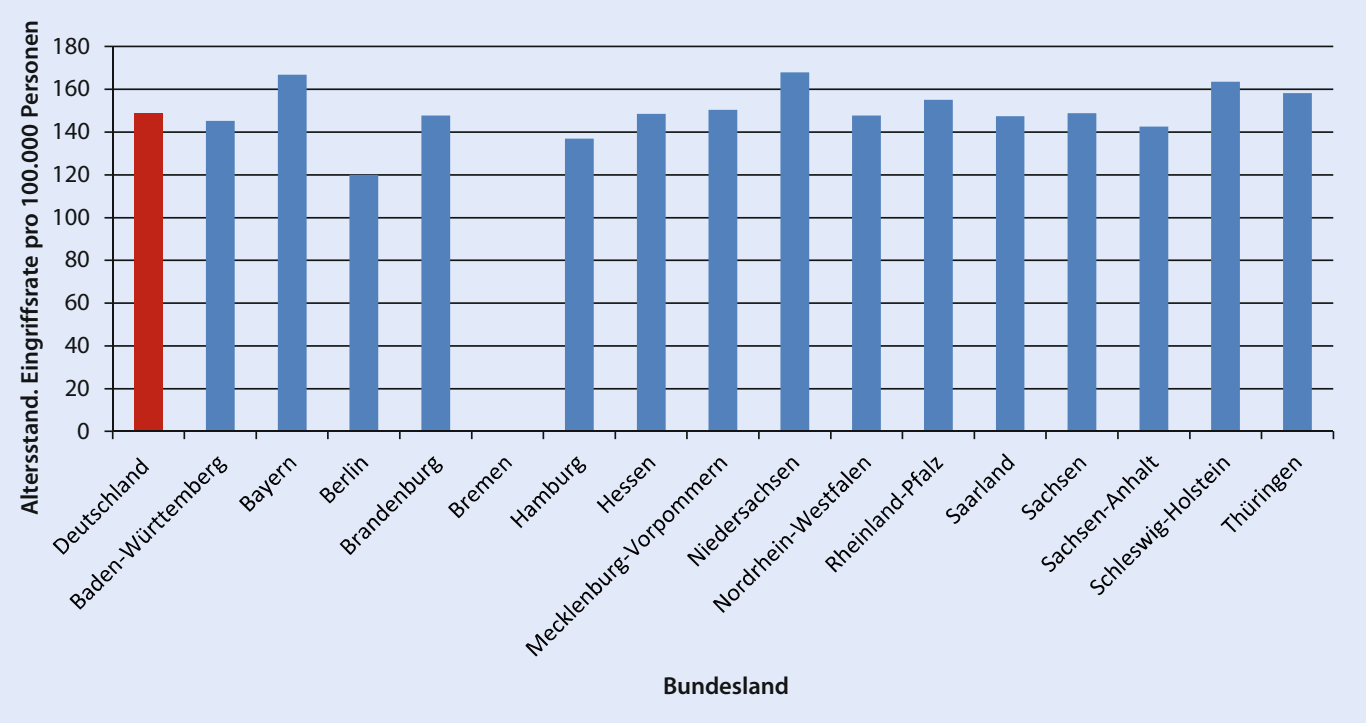

- Abb. 2.6 Altersstandardisierte Eingriffsraten pro 100.000 AOK-Versicherte in 2009, Erstoperation Hüftgelenkersatz. (Quelle: IGES - Schäfer et al. 2013)

liche regionale Unterschiede in der Verteilung in Anspruch genommener Kniegelenkersatzverfahren (- Abb. 2.7). Der Auswertung lagen ebenfalls Abrechnungsdaten von AOK-Versicherten, allerdings aus dem Zeitraum 2005-2011, zugrunde. Auch in dieser Untersuchung waren im Jahr 2011 in Bayern die altersstandardisierten Inanspruchnahmen von Kniegelenkersatzverfahren am höchsten und in Berlin am niedrigsten. Überdurchschnittlich hohe Steigerungsraten von 2005-2011 sind der Berechnung zufolge außerdem an Patienten in den Bundesländern Schleswig-Holstein, Rheinland-Pfalz, Bayern, Thüringen, Hamburg, Hessen und Berlin zu beobachten (Lüring et al. 2013).

Insgesamt wurde für beide Gelenkersatzverfahren eine im Vergleich zum Gesamtdurchschnitt niedrigere Inanspruchnahme in den Regionen Ostdeutschlands festgestellt (mit der Ausnahme von Thüringen) (Schäfer et al. 2013).

Die Inanspruchnahme korrelierte dabei mit der Erkrankungshäufigkeit (Prävalenz) der Arthrose. In Regionen mit einem hohen Vorkommen wurde eine vergleichsweise höhere Inanspruchnahme von Hüftund Knie-TEP beobachtet. Weitere erklärende Variablen für die Inanspruchnahme waren die Facharztdichte (Orthopäden), der regionale sozialökonomi- sche Status und der städtische Raum. Je geringer die regionale Dichte an Orthopäden und je höher der sozioökonomische Status in der Region waren, umso häufiger wurden TEP von Versicherten, die in dieser Region wohnhaft waren, in Anspruch genommen. Im Vergleich zu ländlichen Regionen wurden TEP in den städtischen Regionen deutlich seltener durchgeführt (Schäfer et al. 2013).

- Abb. 2.8 zeigt die von Lüring et al. errechneten altersstandardisierten Eingriffsraten pro 100.000 Einwohner in 2011 für Wechseleingriffe am Knie, unterschieden nach Wohnort-Bundesland der Patienten, sowie den Bundesdurchschnitt als Vergleich. Wechseleingriffe waren dabei definiert als „alle erneuten Operationen am gleichen Kniegelenk«.

Die Analyse zeigt, dass in 2011 bezogen auf die Einwohnerzahl die meisten Wechseleingriffe am Knie bei Patienten in Sachsen-Anhalt, Thüringen, Bayern und Niedersachsen erfolgten. Patienten in Mecklenburg-Vorpommern wiesen die geringste Eingriffsrate auf.

In - Abb. 2.9 wird deutlich, dass die Eingriffsraten in den letzten 10 Jahren teils beachtliche Steigerungen in den Bundesländern aufweisen. Die Abbildung unterscheidet allerdings Steigerungs- 


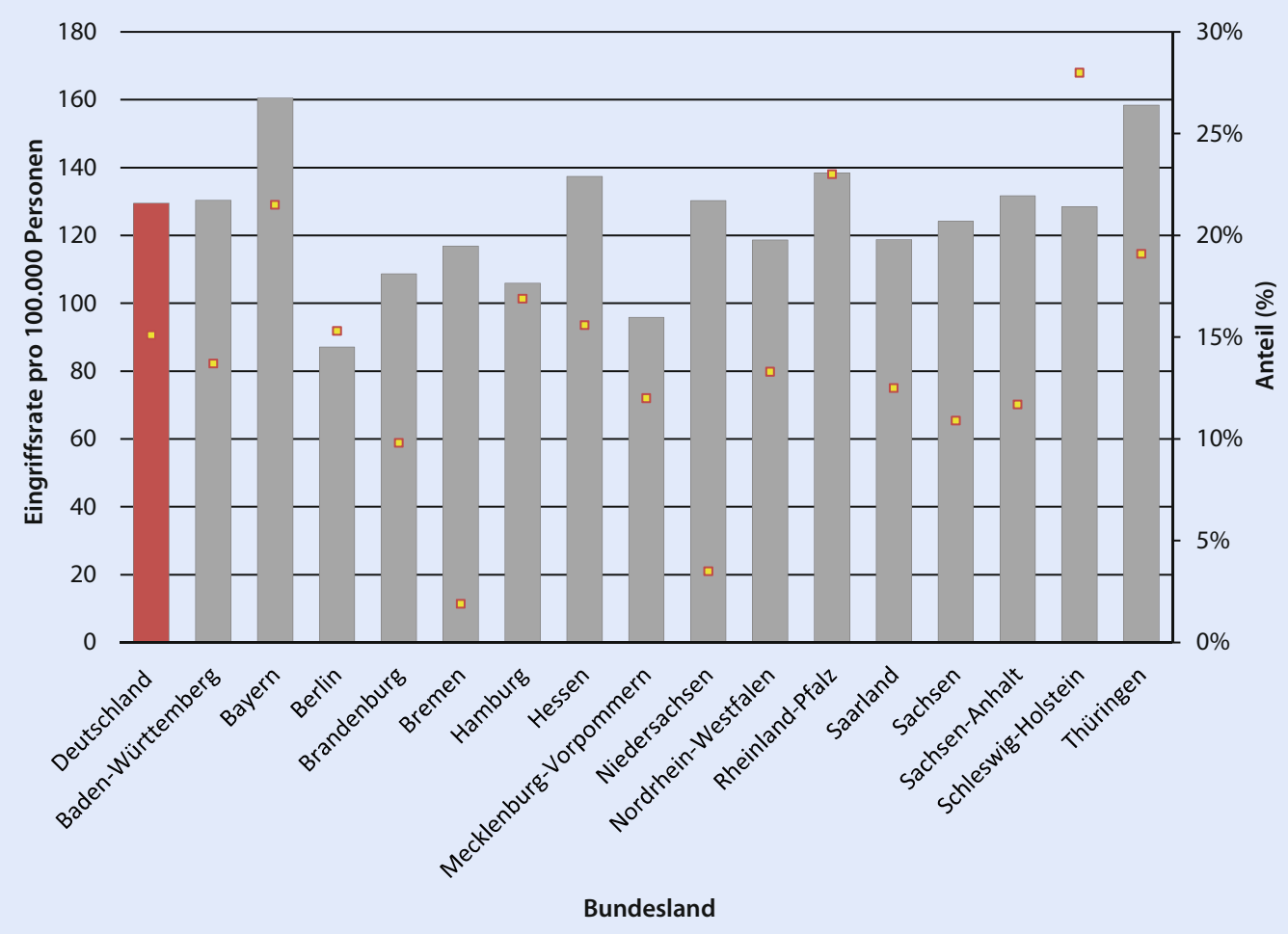

Altersstandardisierte Eingriffsrate pro 100.000 Einwohner, $2011 \quad \square$ Steigerungsrate 2005-2011,\%

- Abb. 2.7 Altersstandardisierte Eingriffsraten pro 100.000 AOK-Versicherte in 2011, Erstimplantationen am Knie, nach Wohnort-Bundesland sowie im Bundesdurchschnitt, mit Steigerungsraten der Eingriffszahlen, 2005-2011. (Quelle: IGES Lüring et al. 2013)

raten zwischen 2005 und 2008 sowie 2008 und 2011, um zu verdeutlichen, dass im früheren Zeitraum der Anstieg der Eingriffsraten im Vergleich zum späteren Zeitraum (mit Ausnahme von Bremen) deutlich größer ausfiel. In den Jahren ab 2008 sind tendenziell niedrigere Steigerungsraten $\mathrm{zu}$ sehen und in einigen Bundesländern sogar rückläufige Raten (Lüring et al. 2013).

Südöstlich gelegene Bundesländer zeigen damit fast durchgängig höhere Operationsraten als jene im Nordosten. Auf Kreisebene werden noch stärkere Unterschiede beobachtet. Bei Primärimplantation wird im Landkreis mit der höchsten Implantationsrate 2,9-mal häufiger ein künstliches Knie implantiert als im Kreis mit der niedrigsten Rate. Für die Revision beträgt die größte Variation zwischen zwei Kreisen maximal das 4,9-Fache (Lüring et al. 2013).
Es werden vielfältige Gründe für die Unterschiede in der Häufigkeit der Versorgung diskutiert: Ein regional unterschiedlicher Zugang zur Krankenhausversorgung wird in Betracht gezogen, ebenso erfolgt eine Verzerrung durch ein Auseinanderfallen von Wohnort und Operationsort. Auch Unterschiede in der Erlösstruktur sowie Fehlanreize durch das Vergütungssystem stehen in der Diskussion, regionale Unterschiede zu begünstigen. Die Autoren verweisen jedoch ausdrücklich auf die Position, dass in der Vergangenheit beobachtete Fallzahlsteigerungen, die nicht auf demographische Verschiebungen zurückzuführen sind, nicht alleine auf monetäre Fehlanreize reduziert werden sollten (Lüring et al. 2013). Insgesamt ist die Datengrundlage aber nicht ausreichend, kausale Zusammenhänge herzustellen (Lüring et al. 2013). 


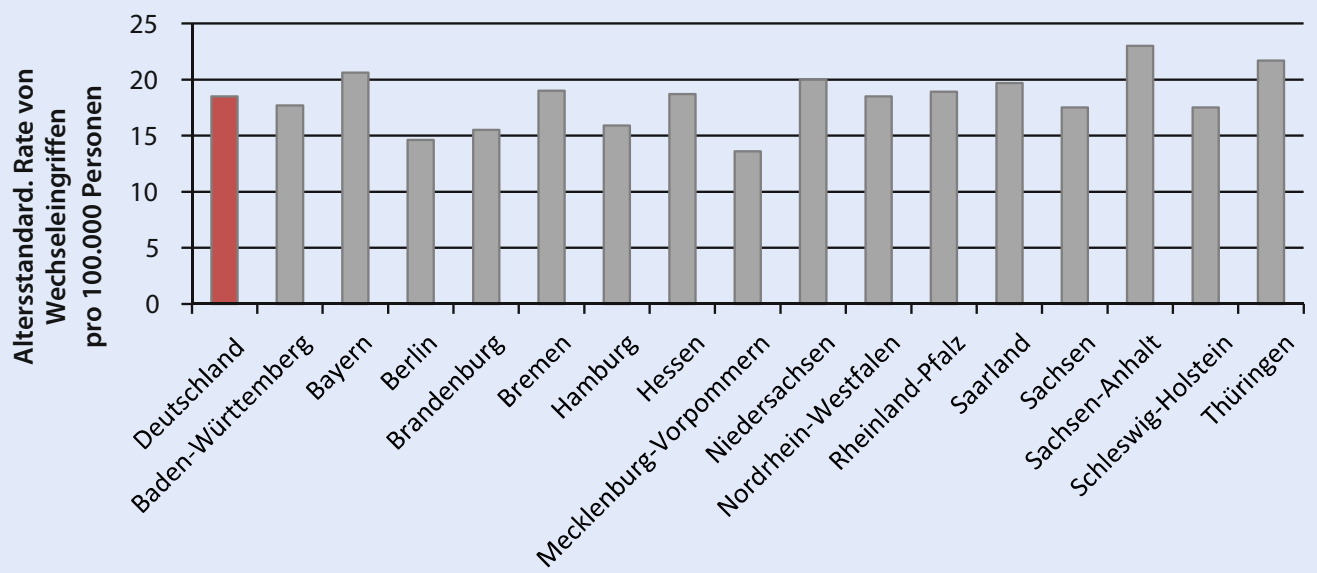

Bundesland

- Abb. 2.8 Altersstandardisierte Eingriffsraten pro 100.000 Einwohner für Wechseleingriffe am Knie, nach Wohnort-Bundesland sowie im Bundesdurchschnitt (2011). (Quelle: IGES - Lüring et al. 2013)

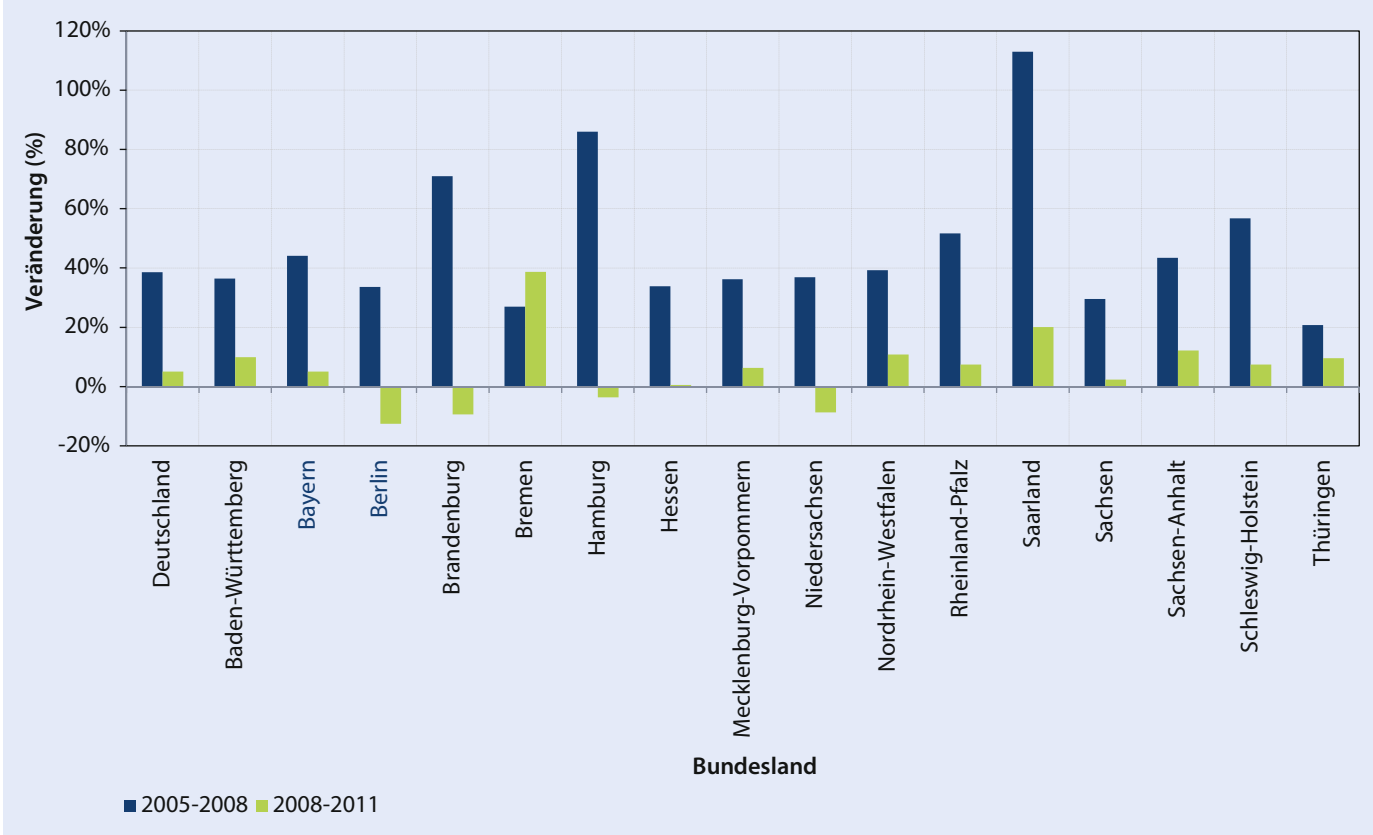

- Abb. 2.9 Veränderungsraten der altersstandardisierten Wechseleingriffsraten (Knie), 2005-2008 und 2008-2011.

(Quelle: IGES - Lüring et al. 2013) 


\subsection{Mengenentwicklung}

\subsubsection{Ersteingriffe}

Seit 2007 nimmt die absolute Anzahl endoprothetischer Hüft- und Knieersteingriffe entsprechend der Zunahme der Anzahl von Personen im höheren Alter (Risikopopulation) in der Bevölkerung zu. Die OPHäufigkeit für Hüft- und Knieersteingriffe hat im Zeitraum 2007 bis 2014 unter Patienten über 70 Jahre innerhalb der Bevölkerung (Bevölkerungsstand des entsprechenden Jahres mit Stichtag 31.12.) über 70 Jahre nicht zugenommen, sondern lag stabil für Hüftersteingriffe bei 1,1\% (2007 und 2014) bzw. bei Knieersteingriffen zwischen 0,7\% (2007) und 0,6\% (2014) (• Abb. 2.10) (eigene Berechnung, Statistisches Bundesamt 2014, Statistisches Bundesamt 2015). Nach einer Zunahme der absoluten Anzahl der Ersteingriffe (2007-2011), war im Bereich des Hüftgelenkersatzes ein leichter Rückgang von 213.935 Fällen in 2011 auf 210.384 Fälle im Jahr 2013 zu verzeichnen, gefolgt von einem Anstieg auf 219.325 Fäl- le in 2014. Im Jahr 2009 wurde mit 159.137 KnieErstimplantationen ein Niveau erreicht, das sich in den Jahren 2010 und 2011 kaum veränderte, sich aber danach verringerte. Im Jahr 2013 wurden 7,6\% weniger Ersteingriffe am Knie durchgeführt als 2008 bzw. 10,1\% weniger Ersteingriffe (absolut Anzahl) als zur Zeit des Höchststandes 2009.

Betrachtet man die Inanspruchnahme von HüftTEP differenziert nach Auswahl der Verankerung, sind Mengenveränderungen im Zeitverlauf erkennbar. Die zementfreie Implantation einer TEP (keine Sonderanfertigung) nahm im beobachteten 6-Jahreszeitraum absolut um $5 \%$ zu. Die Anwendung von zementierten Verfahren ging im gleichen Zeitraum zurück: Zementierte bzw. teilzementierte TEP wurden im Jahre 2013 zu 33\% bzw. 9\% seltener eingesetzt, als es noch 2008 der Fall war. Sonderprothesen nehmen nur eine untergeordnete Rolle ein (- Abb. 2.11).

Die Mengenentwicklung der vier häufigsten Ersteingriffe beim Kniegelenkersatz ist in den letzten Jahren rückläufig (• Abb. 2.12). Der Rückgang

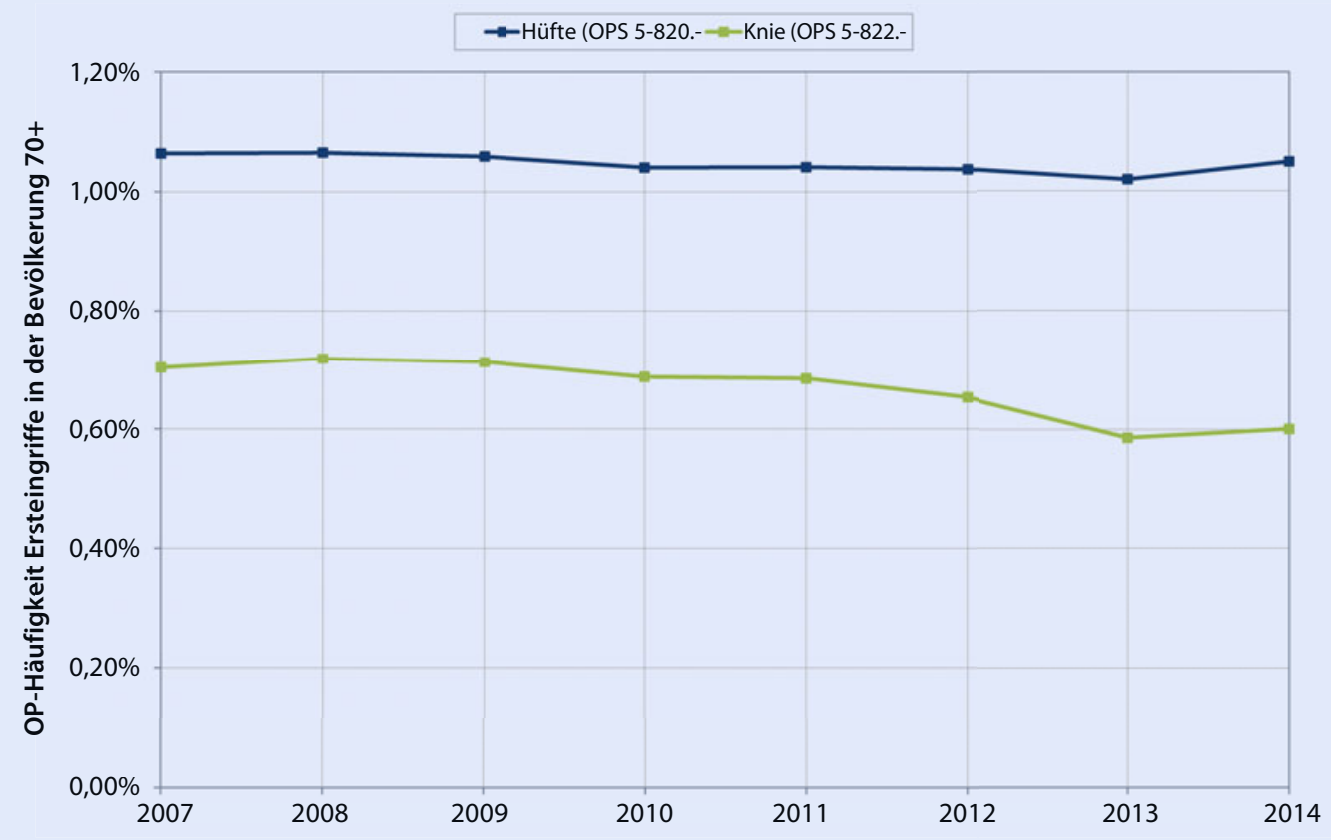

- Abb. 2.10 OP-Häufigkeit Hüft- und Knieersteingriffe in der Bevölkerung 70+ im Zeitverlauf (2007-2014). (Quelle: IGES eigene Berechnung, Statistisches Bundesamt 2014, Statistisches Bundesamt 2015) 


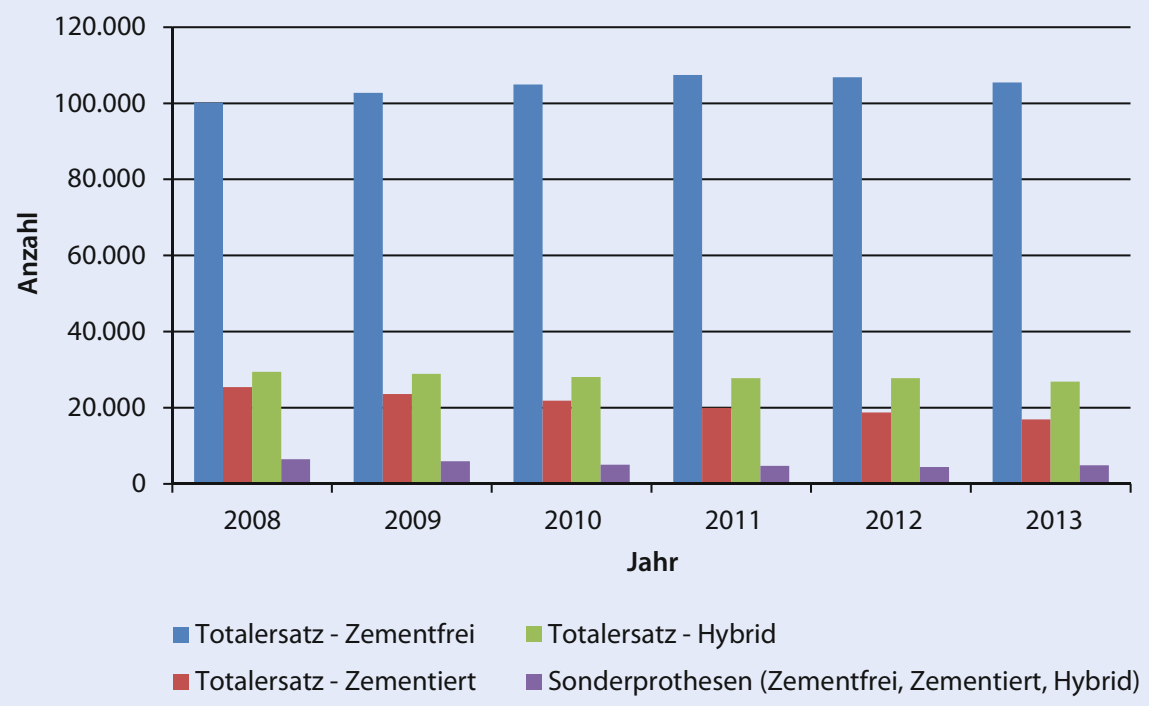

- Abb. 2.11 Absolute Anzahl durchgeführter Hüft-TEP (Ersteingriffe) differenziert nach Verankerung im Zeitverlauf (20082013). (Quelle: IGES - Statistisches Bundesamt 2014)

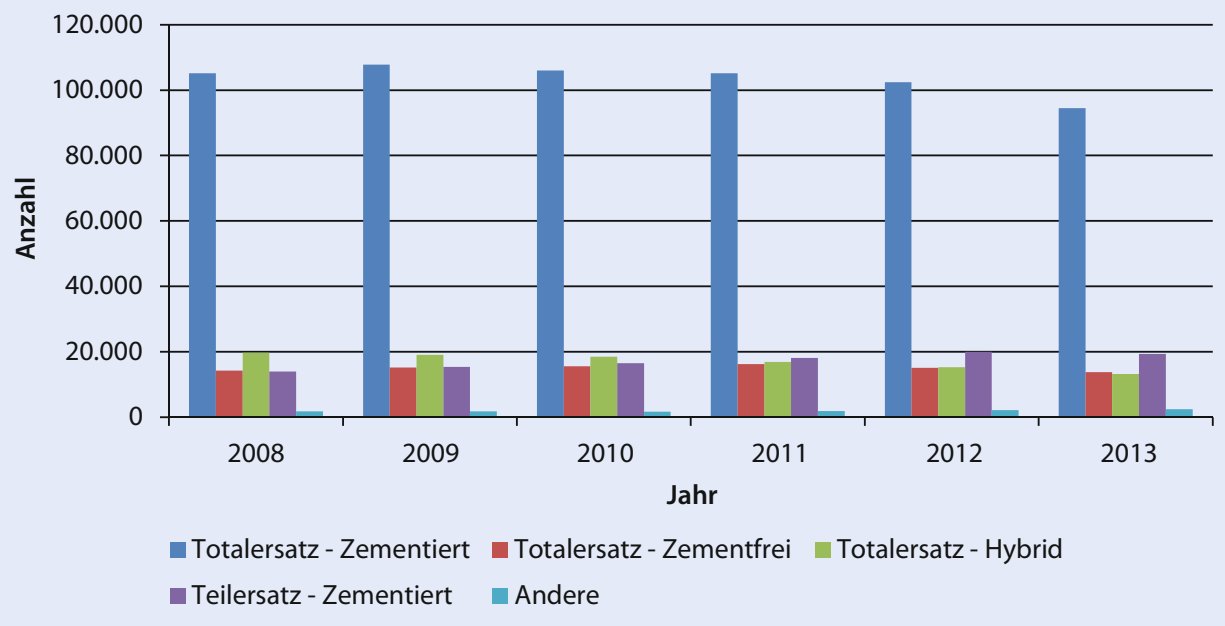

- Abb. 2.12 Absolute Anzahl durchgeführter Knieersteingriffe differenziert nach Verankerung im Zeitverlauf (2008-2013). (Quelle: IGES - Statistisches Bundesamt 2014)

an Ersteingriffen ist primär auf die Verminderung der Inanspruchnahme zementierter Totalersatzeingriffe zurückzuführen.

Eine Auswertung der Entwicklung der Fallzahlen von Hüft- und Knieeingriffen in Deutschland für den Zeitraum 2005-2011 zeigte, dass die Men- genzunahme endoprothetischer Hüftersteingriffe größtenteils durch demographische Veränderungen erklärbar waren, bei der bis 2011 beobachteten $\mathrm{Zu}$ nahme endoprothetischer Knieersteingriffe überwogen hingegen nicht demographische Faktoren (Wengler et al. 2014). 


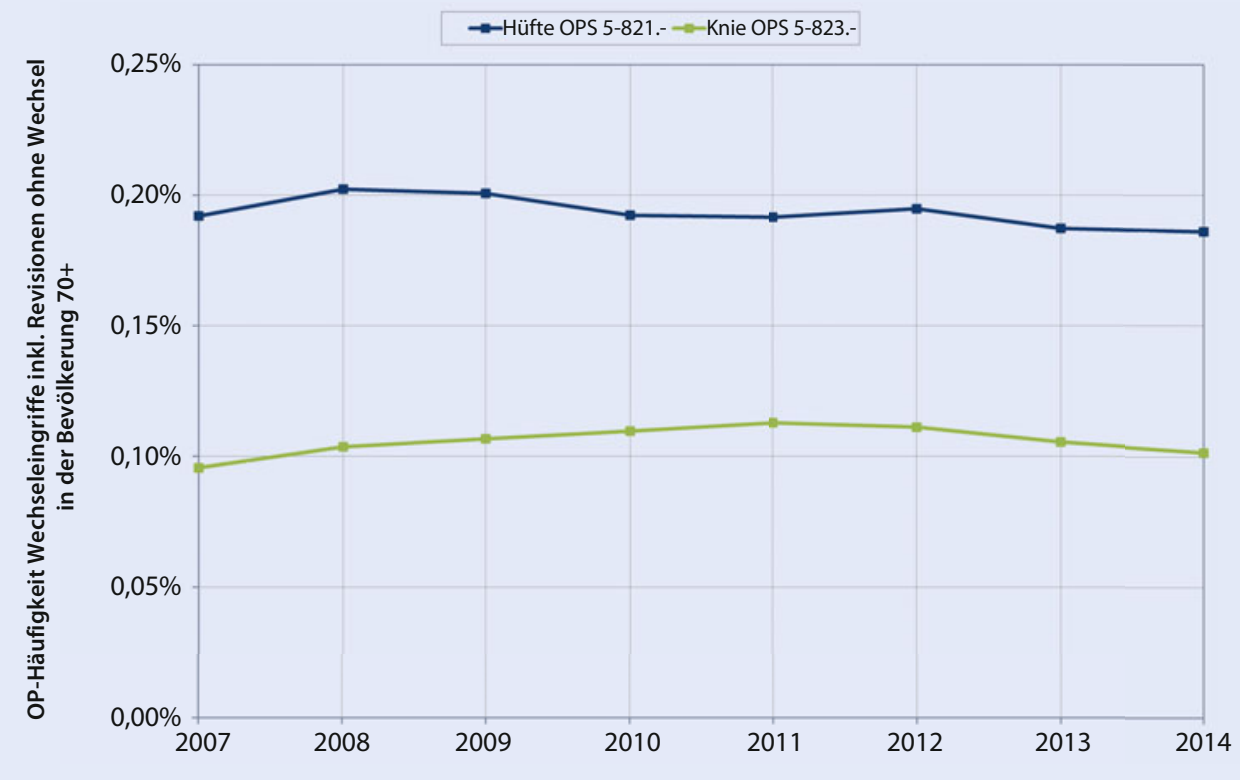

- Abb. 2.13 OP-Häufigkeit Wechseleingriffe und Revisionen (ohne Wechsel) Hüft- und Kniegelenkersatz in der Bevölkerung 70+ im Zeitverlauf (2007-2014). (Quelle: IGES - eigene Berechnung, Statistisches Bundesamt 2014, Statistisches Bundesamt 2015)

Falls sich eine Mengenentwicklung nicht ausreichend durch die demographischen Entwicklungen in der Gesellschaft erklären lässt, kann eine Über- oder Unterversorgung diskutiert werden (Barmer GEK 2010). Neben der Demographie beeinflussen weitere Faktoren und deren Veränderungen (medizinische, ökonomische, systemische, $>$ Abschn. 2.4) die Häufigkeit der Inanspruchnahme medizinischer Leistungen im zeitlichen Verlauf. Deren Effekte können oft nicht ausreichend quantifiziert werden ( $\triangleright$ Kap. 6).

\subsubsection{Wechseleingriffe und Revisionen}

Die absolute Anzahl aller durchgeführter Wechseleingriffe und Revisionen ohne Wechsel an der Hüfte und Knie hat im Zeitraum 2007 bis 2014 zugenommen. Die OP-Häufigkeit unter den über 70-Jährigen hat seit 2007 in der Bevölkerung (Bevölkerungsstand des entsprechenden Jahres, Stichtag 31.12.) weder für Hüft- noch Kniewechseleingriffe (inklusive Revisionen ohne Wechsel) zugenommen und liegt stabil bei Hüftwechseleingriffen bei $0,19 \%$ (2007 und 2014) bzw. für Kniewechseleingriffe bei
0,10\% (• Abb. 2.13) (eigene Berechnung, Statistisches Bundesamt 2014, Statistisches Bundesamt 2015). Die absolute Anzahl der Wechseleingriffe an der Hüfte bezogen auf einen Totalersatz sank im Beobachtungszeitraum 2008-2013 um 12,2\%. Dies ist im Wesentlichen auf einen Rückgang der Eingriffe an zementierten Hüft-TEP zurückzuführen. Diese gingen zwischen 2008 und 2013 kontinuierlich um insgesamt 32,8\% zurück. Dagegen nahm die Anzahl an kodierten Wechseln von nichtzementierten TEP im selben Zeitraum um 8,5\% zu. Die Zunahme ist vermutlich auch auf die höhere Anzahl an eingesetzten zementfreien Implantaten zurückzuführen. Auch teilzementierte TEP und Sonderprothesen wurden 2013 seltener gewechselt als noch 2008. Hier war ein Rückgang von $24,9 \%$ bzw. 17,0 \% zu beobachten. Erfolgt bei einer nichtzementierten TEP ein Wechsel, so wird sie häufig durch eine weitere nichtzementierte TEP (33,2\% der nichtzementierten TEP-Wechsel) oder durch eine Sonderprothese ersetzt $(38,7 \%)$ (• Abb. 2.14).

Der mit Abstand häufigste Wechseleingriff am Knie in den Jahren 2008-2013 ist der Einsatz einer bikondylären Oberflächenersatzprothese, gefolgt 


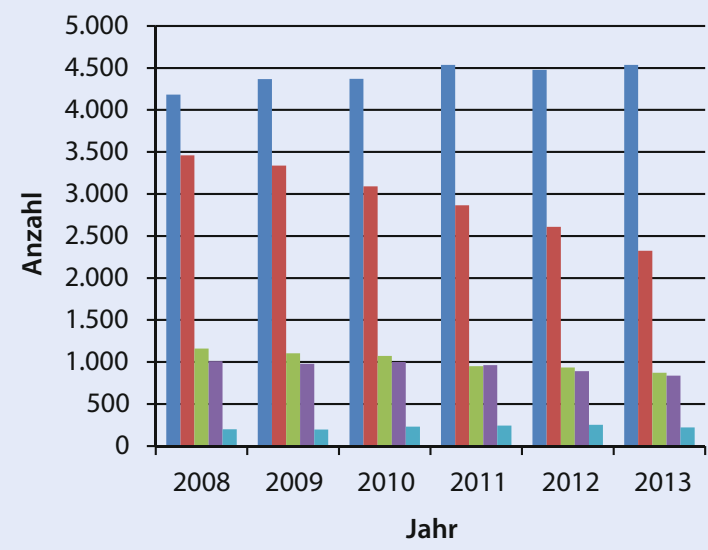

TEP - Zementfrei

- TEP - Zementiert

Hybrid - Teilzementiert

Sonderprothese

Kurzschaft-

Femurkopfprothese

- Abb. 2.14 Absolute Anzahl durchgeführter Hüftwechseleingriffe differenziert nach Verankerung im Zeitverlauf (2008-2013). (Quelle: IGES - Statistisches Bundesamt 2014)

- Tab. 2.3 Absolute Anzahl durchgeführter Wechseleingriffe und Revisionen (ohne Wechsel) am Knie im Zeitverlauf (2008-2013)

\begin{tabular}{|c|c|c|c|c|c|c|c|}
\hline OPS & Bezeichnung & 2008 & 2009 & 2010 & 2011 & 2012 & 2013 \\
\hline $5-823.0$ & Revision (ohne Wechsel) & 3.497 & 3.421 & 3.444 & 3.518 & 3.291 & 3.213 \\
\hline $5-823.1$ & $\begin{array}{l}\text { Wechsel einer unikondylären } \\
\text { Schlittenprothese }\end{array}$ & 1.971 & 1.974 & 2.057 & 2.297 & 2.443 & 2.317 \\
\hline $5-823.2$ & $\begin{array}{l}\text { Wechsel einer bikondylären } \\
\text { Oberflächenersatzprothese }\end{array}$ & 10.590 & 11.049 & 11.821 & 11.916 & 11.614 & 11.290 \\
\hline $5-823.3$ & $\begin{array}{l}\text { Wechsel einer Scharnierendo- } \\
\text { prothese }\end{array}$ & 1.011 & 1.068 & 1.127 & 1.245 & 1.255 & 1.222 \\
\hline $5-823.4$ & Wechsel einer Sonderprothese & 480 & 535 & 529 & 585 & 563 & 533 \\
\hline $5-823.5$ & Wechsel eines Patellaersatzes & 450 & 446 & 535 & 516 & 528 & 439 \\
\hline 5-823.b & $\begin{array}{l}\text { Wechsel einer Endoprothese mit } \\
\text { erweiterter Beugefähigkeit }\end{array}$ & 866 & 811 & 824 & 774 & 840 & 699 \\
\hline $5-823 . c$ & $\begin{array}{l}\text { Wechsel eines interpositionellen } \\
\text { nicht verankerten Implantates }\end{array}$ & 184 & 178 & 174 & 132 & 119 & 100 \\
\hline $5-823 . f$ & $\begin{array}{l}\text { Wechsel einer bikompartimentellen } \\
\text { Teilgelenkersatzprothese }\end{array}$ & 0 & 480 & 512 & 461 & 516 & 459 \\
\hline 5-823.h & $\begin{array}{l}\text { Wechsel eines endoprothetischen } \\
\text { Gelenkersatzes ohne Bewegungs- } \\
\text { funktion }\end{array}$ & 0 & 0 & 0 & 63 & 84 & 112 \\
\hline $5-823 . x$ & Sonstige & 242 & 225 & 241 & 202 & 194 & 188 \\
\hline 5-823.y & N. n. bez. & 31 & 41 & 25 & 19 & 32 & 16 \\
\hline
\end{tabular}


Tab. 2.4 Absolute Anzahl durchgeführter Wechseleingriffe bei bikondylären Oberflächenersatzprothesen im Zeitverlauf (2008-2013)

\begin{tabular}{|c|c|c|c|c|c|c|c|}
\hline OPS & Bezeichnung & 2008 & 2009 & 2010 & 2011 & 2012 & 2013 \\
\hline $5-823.20$ & Typgleich & 305 & 247 & 255 & 228 & 241 & 247 \\
\hline $5-823.21$ & $\begin{array}{l}\text { In eine andere Oberflächenersatz- } \\
\text { prothese, nicht zementiert }\end{array}$ & 47 & 53 & 50 & 31 & 32 & 38 \\
\hline $5-823.22$ & $\begin{array}{l}\text { In eine andere Oberflächenersatz- } \\
\text { prothese, (teil-)zementiert }\end{array}$ & 1.212 & 1224 & 1210 & 1167 & 1116 & 1101 \\
\hline $5-823.23$ & $\begin{array}{l}\text { In eine Scharnierprothese, } \\
\text { nicht zementiert }\end{array}$ & 39 & 58 & 56 & 59 & 67 & 68 \\
\hline $5-823.24$ & $\begin{array}{l}\text { In eine Scharnierprothese, } \\
\text { (teil-)zementiert }\end{array}$ & 2.093 & 2275 & 2474 & 2557 & 2494 & 2362 \\
\hline $5-823.25$ & $\begin{array}{l}\text { In eine Sonderprothese, } \\
\text { nicht zementiert }\end{array}$ & 68 & 71 & 87 & 80 & 84 & 91 \\
\hline $5-823.26$ & $\begin{array}{l}\text { In eine Sonderprothese, } \\
\text { (teil-)zementiert }\end{array}$ & 1.765 & 1938 & 2126 & 2110 & 1927 & 1763 \\
\hline $5-823.27$ & Inlaywechsel & 3.796 & 3961 & 4240 & 4507 & 4539 & 4534 \\
\hline $5-823.28$ & Teilwechsel Femurteil & 287 & 255 & 311 & 257 & 284 & 262 \\
\hline $5-823.29$ & Teilwechsel Tibiateil & 887 & 875 & 934 & 843 & 774 & 738 \\
\hline $5-823.2 x$ & Sonstige & 91 & 92 & 78 & 77 & 56 & 86 \\
\hline
\end{tabular}

von der Revision ohne Wechsel und dem Wechsel einer unikondylären Schlittenprothese (•Tab. 2.3).

Bereits 37,5\% aller betrachteten Wechseleingriffe bei bikondylären Oberflächenersatzprothesen sind durch den Wechsel des Kunststoffinlays erfasst. Dieser Eingriff ist einfacher und komplikationsärmer als der Wechsel anderer, im Knochen verankerter Implantatteile (Lüring et al. 2013). Der Inlaywechsel war bereits 2008 der häufigste Wechseleingriff. Es kam bis 2013 zu einer Zunahme an Inlaywechseln von 19,4\%. Andere zahlenmäßig häufige Eingriffe weisen eine geringere Steigerungsrate auf. Sofern bei einem Wechseleingriff nicht ausschließlich das Inlay gewechselt wird, wird in der Regel der komplette Oberflächenersatz durch zementierte Scharnier- oder Sonderprothesen ersetzt. Andere Verfahren spielen nur eine untergeordnete Rolle. Nur 3,2\% aller Komplett-Endoprothesenwechsel (also ohne Teilwechsel) werden zementfrei durchgeführt (•Tab. 2.4).
Aufgrund der beschriebenen Zunahme der endoprothetischen Knieersteingriffe bis 2009 prognostizierten Lüring et al. (2013) einen entsprechenden Anstieg der Kniewechseloperationen. Die prognostizierte anhaltende Mengenzunahme am Kniegelenkersatz (Haas et al. 2013; Lüring et al. 2013) lässt sich zum heutigen Zeitpunkt auf Basis der OPS-Daten des Statistischen Bundesamtes nicht zeigen (Statistisches Bundesamt 2014).

Pabinger et al. werteten die Inanspruchnahme von Hüftgelenkersatz im Zusammenhang mit ökonomischen Daten der OECD-Länder von 19902011 aus. Sie stellten fest, dass die Zuwachsraten in den Eingriffen besonders stark bei den unter 65-Jährigen ausgeprägt waren und rechnen daher in Zukunft aufgrund des demographischen Wandels mit einer starken Zunahme an Wechsel- und Revisionseingriffen (Pabinger und Geissler 2014). 


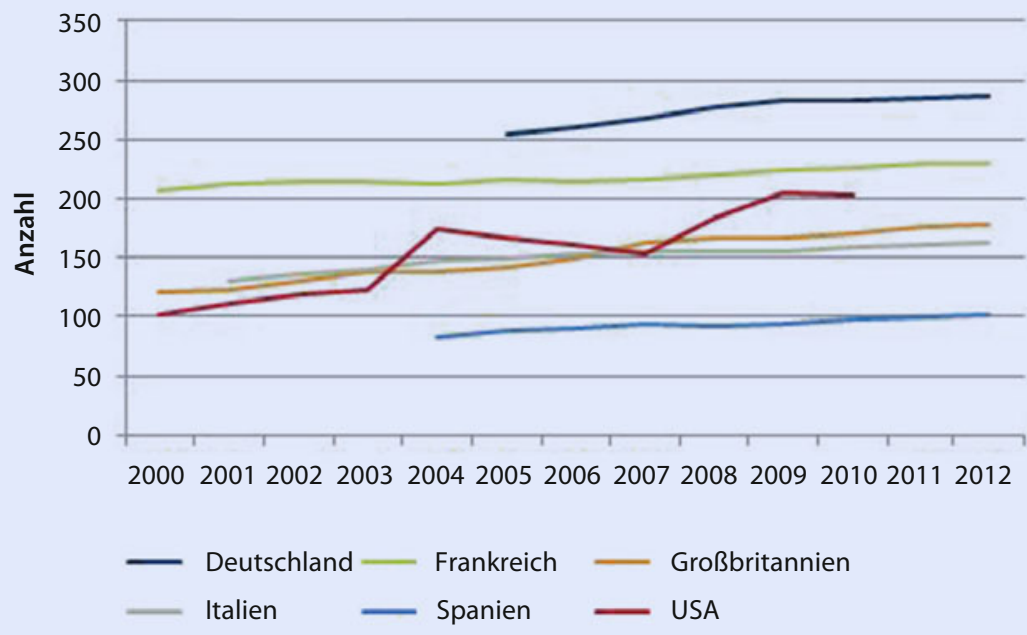

- Abb. 2.15 Internationale Betrachtung der Anzahl endoprothetischer Hüfteingriffe pro 100.000 Einwohner auf Basis von Daten der OECD im Zeitverlauf (2000-2012) (Darstellung von Häufigkeiten ohne Altersadjustierung)* (Quelle: IGES - OECD 2014)

\subsection{Internationaler Vergleich}

Die absolute Anzahl endoprothetischer Hüft- und Knieeingriffe hat in den vergangenen Jahrzehnten nicht nur in Deutschland, sondern europaweit und in den USA zugenommen (Finkenstädt und Niehaus 2015; Merx et al. 2003; Wengler et al. 2014). Mit der Zunahme der Prävalenz altersassoziierter Grunderkrankungen wie Arthrose und Osteoporose, die mit einem erhöhten Risiko für eine Schenkelhalsfraktur einhergehen, und anderer Risikofaktoren für einen Gelenkersatz nimmt auch dessen Bedarf zu (• Abb. 2.15, OECD 2014). Als Grund hierfür werden u. a. der demographische Wandel der Bevölkerung und die damit verbundene Zunahme der Risikopopulation für einen Gelenkersatz genannt (Wengler et al. 2014).

So zeigten Analysen, dass nach Korrektur demographiebedingter Faktoren der Zuwachs endoprothetischer Eingriffe zwischen 2005 und 2011 lediglich $3 \%$ betrug; ohne diese Korrektur betrug der $\mathrm{Zu}$ wachs $11 \%$ (Wengler et al. 2014) ( A Abschn. 2.5.1).

Bei Betrachtung der Anzahl genutzter Gelenkersätze liegt Deutschland im internationalen Vergleich auf Basis von OECD-Angaben in der Spitzengruppe (• Abb. 2.16 u. • Abb. 2.17; OECD 2014). Allerdings sind bei dieser Betrachtung auf Daten- basis der OECD der demographische Wandel, die aktuelle Altersstruktur der Bevölkerungen und weitere die Inanspruchnahme beeinflussende Faktoren nicht berücksichtigt. Aussagen über die länderspezifische Versorgungssituation (Über- oder Unterversorgung), die sich aus diese Daten ableiten, sind auf Grund der starken Altersabhängigkeit endoprothetischer Hüft- und Knieeingriffe allein wegen der fehlenden Berücksichtigung länderspezifischer Altersstrukturen wenig belastbar.

Das Alter und die Altersstrukturen unterscheiden sich international erheblich (• Abb. 2.18). In Deutschland war im Jahr 2012 rund die Hälfte der Bevölkerung 45,53 Jahre alt und älter (Medianalter) und war damit die zweitälteste Bevölkerung nach Japan innerhalb der OECD-Länder. Innerhalb Europas zählt die Bevölkerung Deutschlands zusammen mit Italien zu den ältesten (United Nations 2013). Die Aufteilung der Bevölkerung nach Altersgruppen ist versorgungsrelevant, wenn das Risiko für eine Erkrankung und damit auch die Wahrscheinlichkeit der Inanspruchnahme therapeutischer Maßnahmen, wie bei Gelenkersatz, mit dem Alter deutlich zunimmt.

In einer Studie des Wissenschaftlichen Instituts der Privaten Krankenversicherungen (WIP) wurde der Einfluss des Bevölkerungsalters in verschie- 


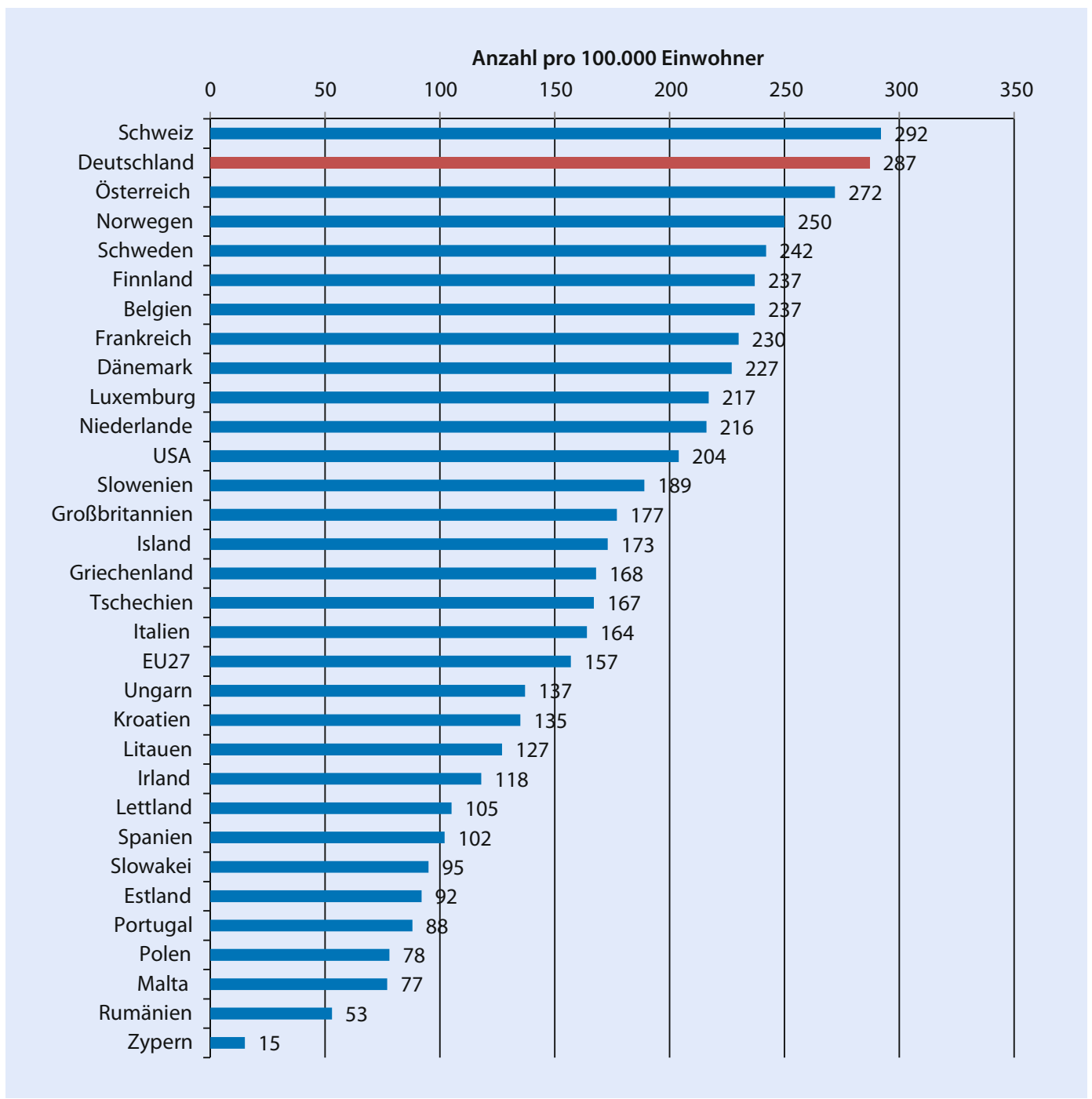

- Abb. 2.16 Inanspruchnahme Hüftgelenkersatz pro 100.000 Einwohner in den OECD-Ländern und den USA, 2012 (oder aktuellste Daten) (Darstellung von Häufigkeiten ohne Altersadjustierung)*. (Quelle: IGES - OECD 2014)

denen Ländern auf die Häufigkeit von 15 chirurgischen Eingriffen inklusive des Gelenkersatzes der Hüfte und des Knies untersucht. Grundlage waren Daten, die in der OECD-Gesundheitsstatistik veröffentlicht wurden (Finkenstädt und Niehaus 2015). Deutschland hatte auf Basis der in die Studie einbezogenen Daten mit einem Medianalter von 44,3 Jahren nach Japan $(44,6)$ unter den betrachteten Ländern das höchste Bevölkerungsalter (• Abb. 2.19).
Finkenstädt et al. zeigten, dass eine Berücksichtigung der Altersstruktur der deutschen Bevölkerung einen Einfluss auf die Rangordnung des internationalen Ländervergleiches (Hüfte: 32 Länder, Knie: 21 Länder) hat. Beim Hüftgelenkersatz nimmt Deutschland nach Berücksichtigung der Altersstruktur Platz 5 anstatt Platz 2 ein und rangiert damit hinter der Schweiz, Norwegen, Österreich und Luxemburg; beim Kniegelenkersatz verschiebt sich die deutsche Rangposition von Platz 5 auf Platz 8 


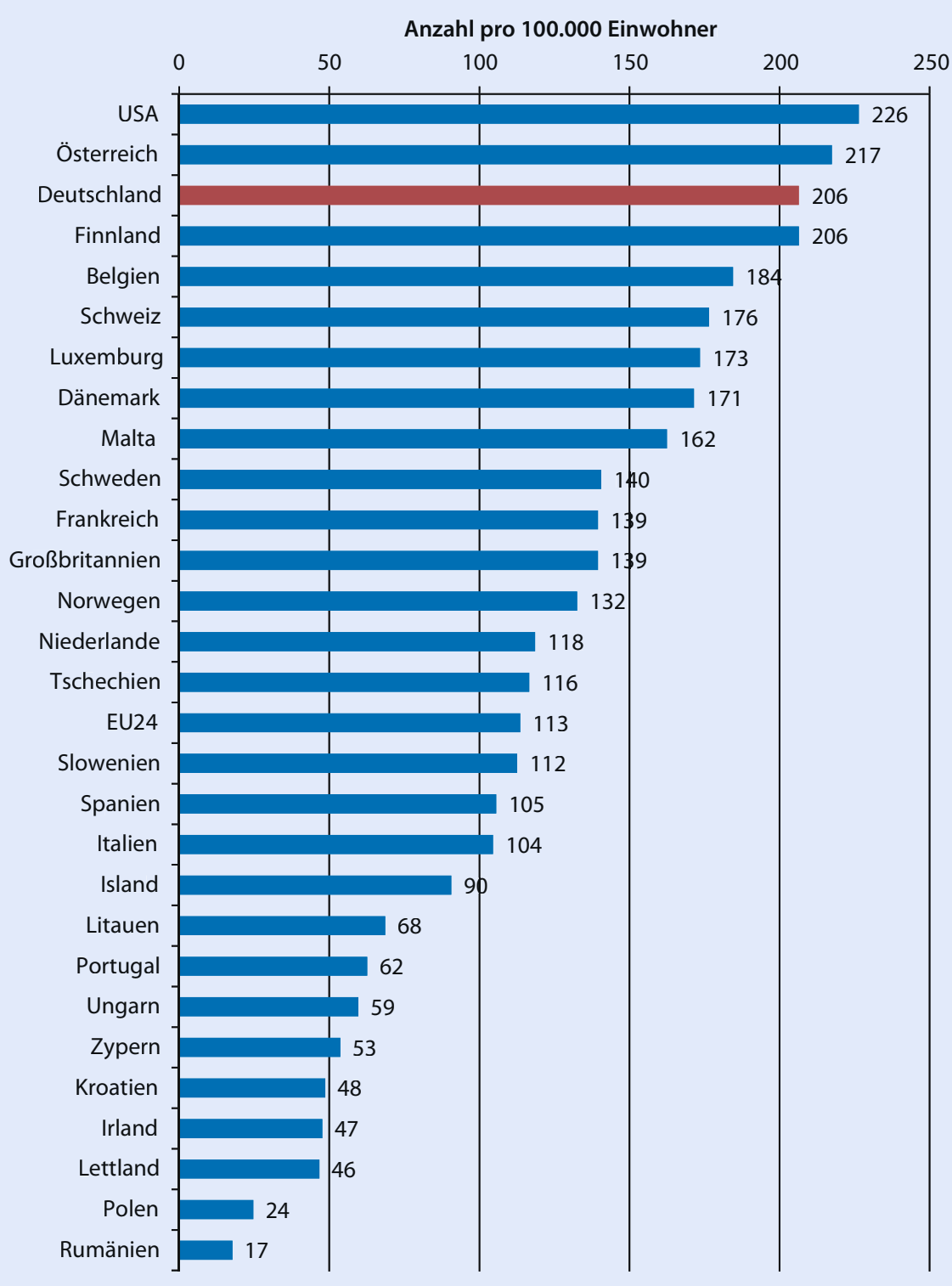

- Abb. 2.17 Inanspruchnahme Kniegelenkersatz pro 100.000 Einwohner in den OECD-Ländern und den USA, 2012 (oder aktuellste Daten) (Darstellung von Häufigkeiten ohne Altersadjustierung)*. (Quelle: IGES - OECD 2014) 


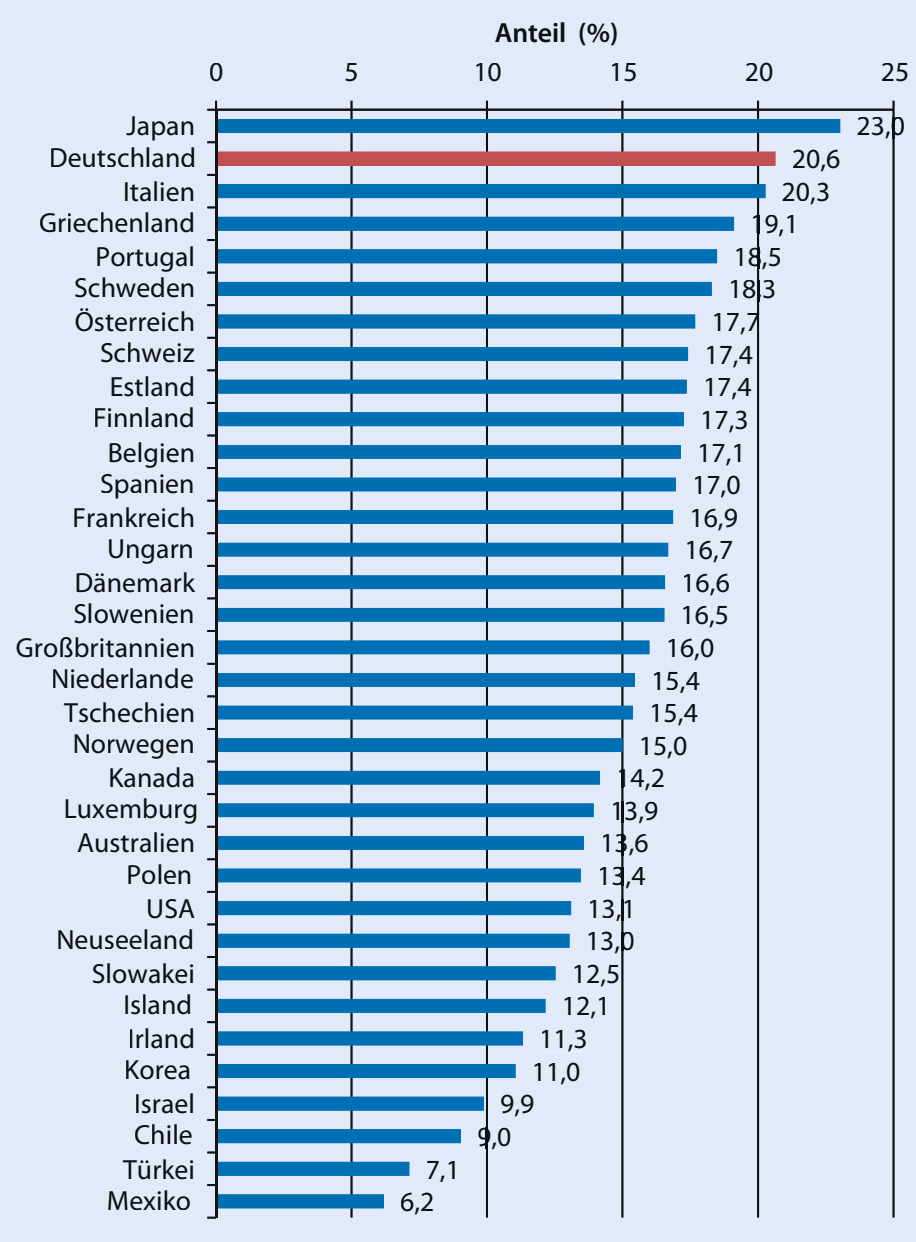

- Abb. 2.18 Anteil der Personen im Alter $\geq 65$ Jahre an der Gesamtbevölkerung, 2010. (Quelle: IGES - OECD 2014)

(• Abb. 2.20 u. - Abb. 2.21; Finkenstädt und Niehaus 2015, 2013). Der Faktor zwischen geringster und höchster OP-Häufigkeit, wird als Indikator für die Versorgungssituation diskutiert (Niethard et al. 2013). Auf Basis der OECD-Daten liegt dieser Faktor für endoprothetische Hüfteingriffe innerhalb Deutschlands bei 2 (Finkenstädt und Niehaus 2015) und innerhalb der USA bei 4 (Fisher et al. 2010). Endoprothetischen Knieeingriffen unterscheiden sich regional innerhalb Deutschlands um den Faktor 3,2 (Finkenstädt und Niehaus 2015) und in den USA um den Faktor 3,8 (Fisher et al. 2010). Insbesondere bei den Hüfteingriffen kann bei einer hohen Rate, aber vergleichsweise geringen regionalen Varianz, von einer allgemein akzeptierten Indikationsstellung und einem akzeptiertem Versorgungsstandard ausgegangen werden (Niethard et al. 2015).

Neben demographischen beeinflussen auch soziale, ökonomische, strukturelle oder medizinische Faktoren (Merx et al. 2003; Pabinger und Geissler 2014) sowie spezifische Charakteristika der nationalen Gesundheitssysteme, wie beispielsweise unterschiedliche Kodiersysteme und Unterschiede in der Datenerfassung, die Inanspruchnahme einer Gesundheitsleistung bzw. deren Abbildung. So melden einige Länder nur den totalen Hüftgelenkersatz (z. B. Estland), andere schließen auch den partiellen Hüftgelenkersatz ein (OECD 2014). In einigen Län- 


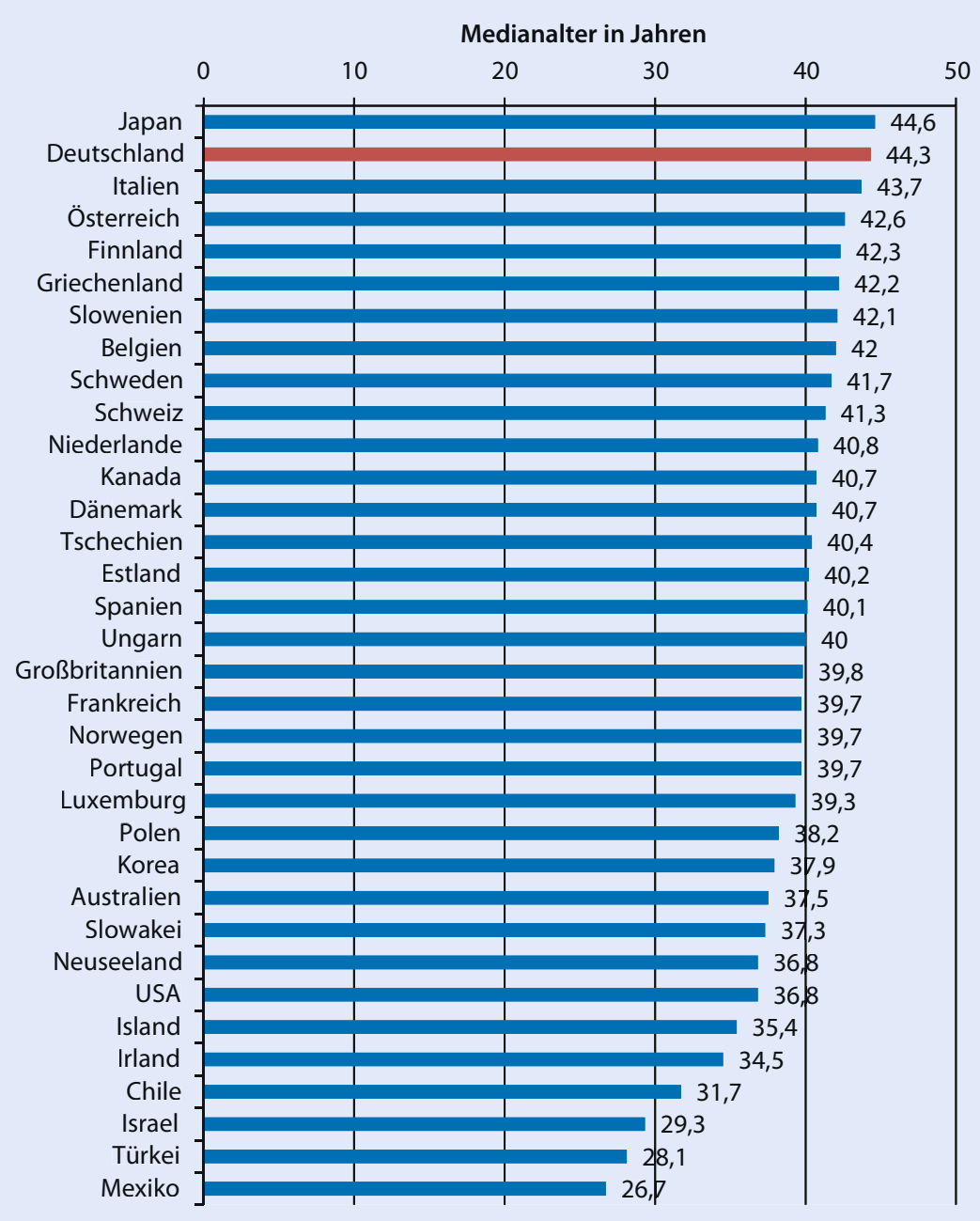

- Abb. 2.19 Medianalter der OECD-Länder, 2010. (Quelle: IGES - OECD 2014, Finkenstädt und Niehaus 2015)

dern fließen Datenmeldungen aus privaten Krankenhäusern nicht (z. B. Irland) oder nur zum Teil (z. B. Spanien) in die Statistiken ein (Finkenstädt und Niehaus 2015; OECD 2014). Die Inanspruchnahme von Gelenkersatzverfahren steht auch mit der wirtschaftlichen Leistung und den Pro-KopfGesundheitsausgaben eines Landes in Zusammenhang (Pabinger und Geissler 2014).
Dies macht deutlich, dass Daten aus internationalen Vergleichen einer vorsichtigen Interpretation bedürfen. Bewertungen der nationalen Versorgungssituation basierend auf internationalen Vergleichen oder Rangbildungen der OECD-Daten ohne entsprechende Adjustierung sind nicht belastbar. 


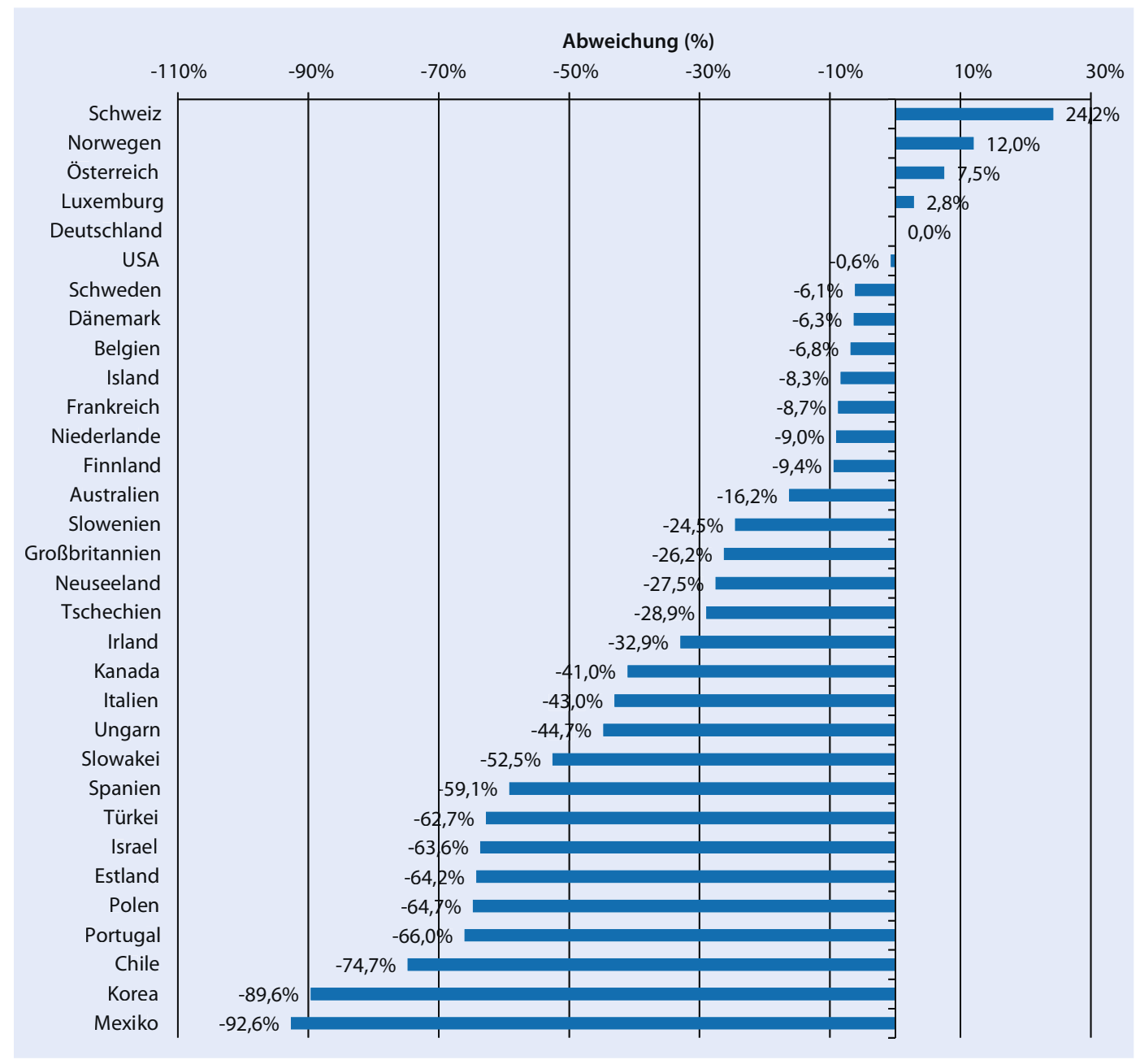

- Abb. 2.20 Abweichung von der deutschen Fallzahl nach Altersstandardisierung bei Hüftgelenkersatzeingriffen. (Quelle: IGES - Finkenstädt und Niehaus 2015) 


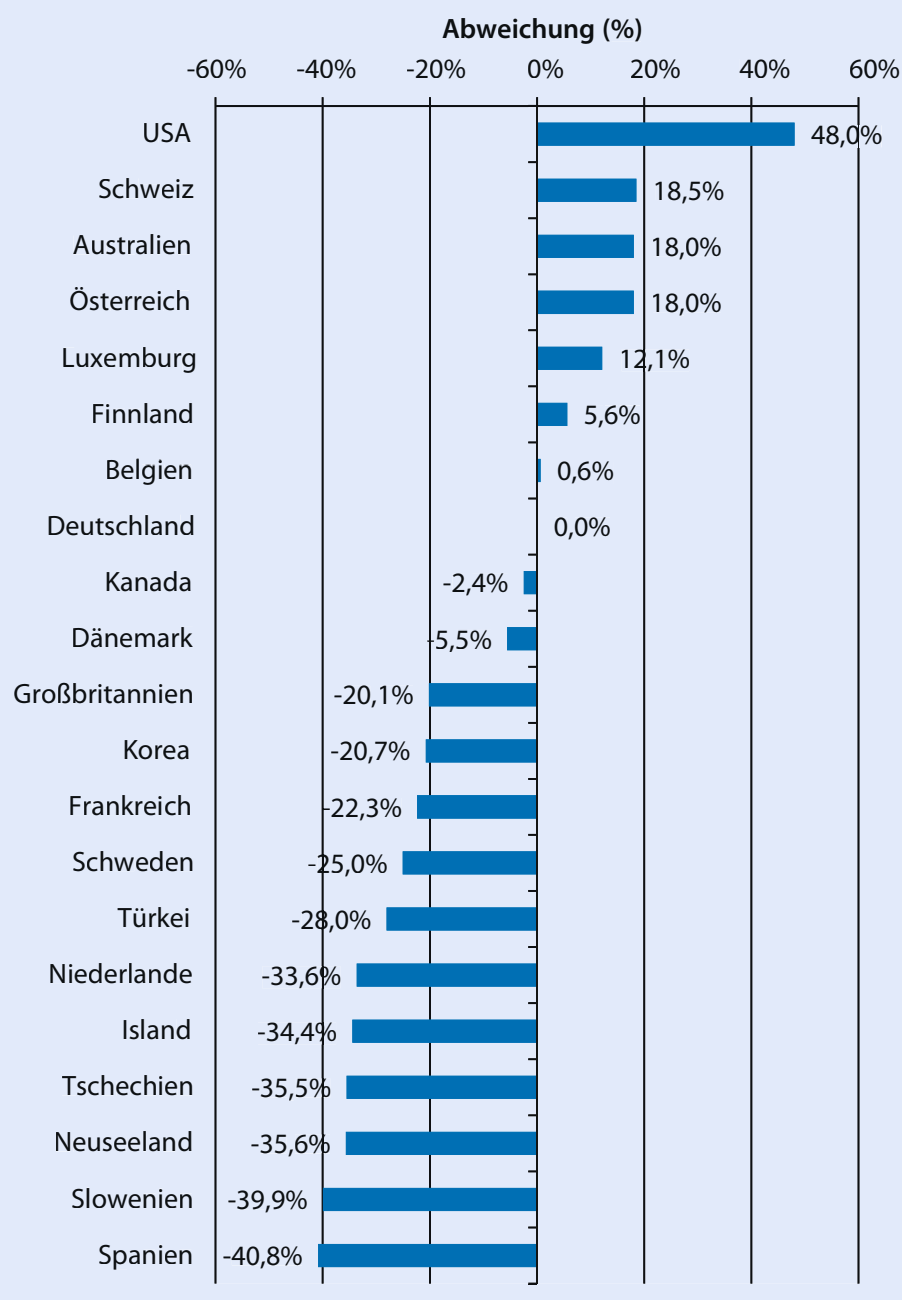

- Abb. 2.21 Abweichung von der deutschen Fallzahl nach Altersstandardisierung bei Kniegelenkersatzeingriffen. (Quelle: IGES - Finkenstädt und Niehaus 2015)

Open Access Dieses Kapitel wird unter der Creative Commons Namensnennung-Nicht kommerziell 4.0 International Lizenz (http://creativecommons.org/licenses/by-nc/4.0/deed.de) veröffentlicht, welche für nicht kommerzielle Zwecke die Nutzung, Vervielfältigung, Bearbeitung, Verbreitung und Wiedergabe in jeglichem Medium und Format erlaubt, sofern Sie den/die ursprünglichen Autor(en) und die Quelle ordnungsgemäß nennen, ein Link zur Creative Commons Lizenz beifügen und angeben, ob Änderungen vorgenommen wurden.

Etwaige Abbildungen oder sonstiges Drittmaterial unterliegen ebenfalls der genannten Creative Commons Lizenz, sofern sich aus der Abbildungslegende oder der Quellreferenz nichts anderes ergibt. Sofern solches Drittmaterial nicht unter der genannten Creative Commons Lizenz steht, ist eine Vervielfältigung, Bearbeitung oder öffentliche Wiedergabe nur mit vorheriger Zustimmung des betreffenden Rechteinhabers oder auf der Grundlage einschlägiger gesetzlicher Erlaubnisvorschriften zulässig. 


\section{Literatur}

BARMER GEK Report Krankenhaus 2010. Schwerpunktthema: Trends in der Endoprothetik des Hüftund Kniegelenks. Schriftenreihe zur Gesundheitsanalyse, Band 3. St. Augustin: Asgard-Verlag. ISBN: 978-537-44103-4.

Braun B (2013): Knie- und Hüft-(Total-) Endoprothesen 2008 bis 2012 - hkk Gesundheitsreport. Bremen: hkk Erste Gesundheit. https://www.hkk.de/fileadmin/doc/broschueren_flyer/sonstiges/20131129_hkk_Gesundheitsreport_Knie-Hueft-Tep.pdf. [Abruf am: 03.11.2015].

DIMDI (2015): Operationen an den Bewegungsorganen (5-78...5-86). OPS Version 2015. Letzte Aktualisierung: 17. Oktober 2014. Köln: Deutsches Institut für Medizinische Dokumentation und Information. https://www.dimdi.de/static/de/klassi/ops/kodesuche/ onlinefassungen/opshtml2015/block-5-78...5-86.htm [Abruf am: 23.06.2015].

Finkenstädt V \& Niehaus F (2013): Rationierung und Versorgungsunterschiede in Gesundheitssystemen. Ein internationaler Überblick. Köln: Wissenschaftliches Institut der PKV. ISBN: 978-3-9813569-4-6.

Finkenstädt V \& Niehaus F (2015): Die Aussagekraft von Länderrankings im Gesundheitsbereich. Köln: Wissenschaftliches Institut der PKV. ISBN: 978-3-9813569-7-7.

Fisher E, Bell J, Tomek I, Esty A \& Goodman D (2010): Trends and regional variation in hip, knee, and shoulder replacement. http://www.dartmouthatlas.org/downloads/ reports/Joint_Replacement_0410.pdf [Abruf 06.06.2015].

Haas H, Grifka J, Günther KP, Heller KD, Niethard FU, Windhagen $\mathrm{H}$, Ebner M \& Mittelmeier W (2013): EndoCert. Zertifizierung von Endoprothetischen Versorgungszentren in Deutschland. Stuttgart: Georg Thieme Verlag KG. ISBN: 978-3-13-174081-6.

Lüring C, Niethard FU, Günther KP, Schäfer T, Hannemann F, Pritzkuleit R, Meier W \& Kirschner S (2013): Regionale Unterschiede und deren Einflussfaktoren - Schwerpunkt Knieendoprothetik. Report der Deutschen Gesellschaft für Orthopädie und Orthopädische Chirurgie. Bertelsmann Stiftung.

Merx H, Dreinhofer K, Schrader P, Sturmer T, Puhl W, Gunther KP \& Brenner H (2003): International variation in hip replacement rates. Annals of the rheumatic diseases 62(3), 222-226. ISSN: 0003-4967.

Niethard F, Mahlzahn J, Schäfer T (2013): Endoprothetik und Wirbelsäuleneingriffe - Uneinheitliches Versorgungsgeschehen. Deutsches Ärzteblatt 110(27-28), 1362-1365.

OECD (2014): Health at a Glance: Europe 2014. OECD Publishing. ISBN: 978-92-64-22327-1.

Pabinger C \& Geissler A (2014): Utilization rates of hip arthroplasty in OECD countries. Osteoarthritis Cartilage 22(6), 734-741. DOI: S1063-4584(14)01044-9 pii ;10.1016/j. joca.2014.04.009.

Rabenberg M (2013): Arthrose. Gesundheitsberichterstattung des Bundes. Heft 54. Berlin: Robert Koch-Institut, Statistisches Bundesamt. ISBN: 978-3-89606-219-2.
Schäfer T, Pritzkuleit R, Jeszenszky C, Malzahn J, Maier W, Gunther KP \& Niethard F (2013): Trends and geographical variation of primary hip and knee joint replacement in Germany. Osteoarthritis and Cartilage 21(2), 279-288. DOI: 10.1016/j.joca.2012.11.006.

Statistisches Bundesamt (2014): Gesundheit. Fallpauschalenbezogene Krankenhausstatistik (DRG-Statistik) Operationen und Prozeduren der vollstationären Patientinnen und Patienten in Krankenhäusern - Ausführliche Darstellung - 2013. Wiesbaden.

Statistisches Bundesamt (2015): https://www.destatis.de/DE/ ZahlenFakten/GesellschaftStaat/Bevoelkerung/Bevoelkerung.html [Abruf am: 31.5.2016].

United Nations (2013): World Population Prospects: The 2012 Revision, Highlights and Advance Tables. Working Paper No. ESA/P/WP.228. 2015/12/29/. United Nations, Department of Economic and Social Affairs, Population Division. http://esa.un.org/unpd/wpp/Publications/Files/ WPP2012_HIGHLIGHTS.pdf [Abruf am: 04.11.2015].

Wengler A, Nimptsch U \& Mansky T (2014): Hip and knee replacement in Germany and the USA: analysis of individual inpatient data from German and US hospitals for the years 2005 to 2011. Deutsches Arzteblatt international 111(23-24), 407-416. DOI: 10.3238/arztebl.2014.0407. 